\title{
A New Probability Distribution for Modeling Failure and Service Times: Properties, Copulas and Various Estimation Methods
}

\author{
Hanaa Elgohari $^{1, *}$, Mohamed Ibrahim ${ }^{2}$, Haitham M. Yousof ${ }^{3}$ \\ ${ }^{1}$ Department of applied statistics, Faculty of commerce, Mansoura University, Egypt \\ ${ }^{2}$ Department of Applied, Mathematical and Actuarial Statistics, Faculty of Commerce, Damietta University, Damietta, Egypt \\ ${ }^{3}$ Department of Statistics, Mathematics and Insurance, Benha University, Benha, Egypt
}

\begin{abstract}
In this paper, a new generalization of the Pareto type II model is introduced and studied. The new density can be "right skewed" with heavy tail shape and its corresponding failure rate can be "J-shape", "decreasing" and "upside down (or increasing-constant-decreasing)". The new model may be used as an "under-dispersed" and "over-dispersed" model. Bayesian and non-Bayesian estimation methods are considered. We assessed the performance of all methods via simulation study. Bayesian and non-Bayesian estimation methods are compared in modeling real data via two applications. In modeling real data, the maximum likelihood method is the best estimation method. So, we used it in comparing competitive models. Before using the the maximum likelihood method, we performed simulation experiments to assess the finite sample behavior of it using the biases and mean squared errors.
\end{abstract}

Keywords Pareto type II Distribution, Estimation, Simulations, Copulas, Renyi Copula, Farlie Gumbel Morgenstern Family, Ordinary Least Square, Bayesian Estimation.

AMS 2010 subject classifications 62E10, 62N01, 62N02

DOI:10.19139/soic-2310-5070-1101

\section{Introduction}

The Pareto type II (also called as Lomax) distribution is a heavy-tail probability distribution used in business, actuarial science, biological sciences, engineering, economics, income and wealth inequality, queueing theory, size of cities, and Internet traffic modeling (see Lomax [41]). It has been applied to model data obtained from income and wealth (Harris [34] and Atkinson and Harrison [10]), firm size (Corbellini et al. [31]) and reliability and life testing (Hassan and Al-Ghamdi [35]). The Pareto type II model is known as a special model form of Pearson type VI distribution and has also considered as a mixture of exponential (Exp) and gamma (Gam) distributions. The Pareto type II model belongs to the family of "decreasing" hazard rate function (HRF) and considered as a limiting model of residual lifetimes at great age (Balkema and de Hann [19] and Chahkandi and Ganjali [21]). The Pareto type II distribution has been suggested as heavy tailed alternative to the Exp, Weibull (W) and Gam distributions (Bryson [20]). For details about relation between the Pareto type II model and the Burr family and Compound Gamma (CGam) model see Tadikamalla [62] and Durbey [24].

The main aim of this work is to provide a flexible extension of the Pareto type II distribution using the odd Burr$\mathrm{G}(\mathrm{OB}-\mathrm{G})$ family defined by Alizadeh et al. [6]. Bayesian and Non-Bayesian estimation methods such as uch as the maximum likelihood estimation (MLE), ordinary least square estimation (OLSE), weighted least square estimation

\footnotetext{
*Correspondence to: Hanaa Elgohari (Email: hanaa_elgohary@mans.edu.eg). Department of applied statistics, Faculty of commerce, Mansoura University, Egypt.
}

ISSN 2310-5070 (online) ISSN 2311-004X (print)

Copyright (C) 2021 International Academic Press 
(WLSE) and the Kolmogorov estimation (KE) are considered. The Bayesian estimation method under the squared error loss function (SELF). The new model proved its ability in modeling

- The monotonically increasing hazard rate data.

- The bimodal left skewed real data sets.

- The bimodal right skewed real data sets.

A random variable (rv) $Z$ has the Pareto type II (P) distribution with two parameters $\alpha$ and $\beta$ if it has cumulative distribution function (CDF) (for $Z>0$ ) given by

$$
G_{\alpha, \beta}(\mathbf{z})=1-\left(1+\frac{\mathbf{z}}{\beta}\right)^{-\alpha}
$$

where $\alpha>0$ and $\beta>0$ are the shape and scale parameters, respectively. Then the corresponding probability density function (PDF) of (1) is

$$
g_{\alpha, \beta}(\mathbf{z})=\frac{\alpha}{\beta}\left(1+\frac{\mathbf{z}}{\beta}\right)^{-(\alpha+1)} .
$$

Due to Alizadeh et al. [6], the CDF of the OB-G family is given by

$$
F_{v, \theta, \underline{\zeta}}(\mathbf{z})=1-\frac{\bar{G}_{\underline{\zeta}}(\mathbf{z})^{v \theta}}{\left[G_{\underline{\zeta}}(\mathbf{z})^{v}+\bar{G}_{\underline{\zeta}}(\mathbf{z})^{v}\right]^{\theta}},
$$

where $\bar{G}_{\underline{\zeta}}(\mathbf{z})=1-G_{\underline{\zeta}}(\mathbf{z})$. The PDF corresponding to (3) is given by

$$
f_{v, \theta, \underline{\zeta}}(\mathbf{z})=\frac{v \theta g_{\underline{\zeta}}(\mathbf{z}) G_{\underline{\zeta}}(\mathbf{z})^{v-1} \bar{G}_{\underline{\zeta}}(\mathbf{z})^{v \theta-1}}{\left[G_{\underline{\zeta}}(\mathbf{z})^{v}+\bar{G}_{\underline{\zeta}}(\mathbf{z})^{v}\right]^{1+\theta}} .
$$

For $\theta=1$, the OB-G family reduces to the Odd G (O-G) family (see Gleaton and Lynch [42]). For $v=1$, the OB-G family reduces to the proportional reversed hazard rate (PRHR) family. The odd Burr Pareto type II (OBP) survival function (SF) is given by

$$
\left.S_{\underline{\xi}}(\mathbf{z})\right|_{(\underline{\xi}=v, \theta, \alpha, \beta)}=\frac{\nabla_{\beta}(\mathbf{z})^{-\alpha v \theta}}{\left\{\left[1-\nabla_{\beta}(\mathbf{z})^{-\alpha}\right]^{v}+\nabla_{\beta}(\mathbf{z})^{-\alpha v}\right\}^{\theta}},
$$

where $\nabla_{\beta}(\mathbf{z})=1+\frac{\mathbf{z}}{\beta}$ and $S_{\underline{\xi}}(\mathbf{z})=1-\left.F_{\underline{\xi}}(\mathbf{z})\right|_{(\underline{\xi}=v, \theta, \alpha, \beta)}$. For $\theta=1$, the OBP reduces to the odd Pareto type II. For $v=1$, the OBP reduces to the proportional reversed hazard rate Pareto type II. The PDF corresponding to (5) is given by

$$
f_{\underline{\xi}}(\mathbf{z})=v \theta \frac{\alpha}{\beta} \frac{\nabla_{\beta}(\mathbf{z})^{-(\alpha v \theta+1)}\left[1-\nabla_{\beta}(\mathbf{z})^{-\alpha}\right]^{v-1}}{\left\{\left[1-\nabla_{\beta}(\mathbf{z})^{-\alpha}\right]^{v}+\nabla_{\beta}(\mathbf{z})^{-\alpha v \theta}\right\}^{1+\theta}} .
$$

The HRF for the new model can be derived from $f_{\underline{\xi}}(\mathbf{z}) / S_{\underline{\xi}}(\mathbf{z})$. Many useful Pareto type II extensions can be found in Tahir et al. [64] (Weibull Pareto type II distribution), Cordeiro et al. [23] (the one parameter Pareto type II system of densities), Altun et al. [12] (Odd log-logistic Pareto type II), Altun et al. [12] (ZografosBalakrishnan Pareto type II distribution), Elbiely and Yousof [33] (Weibull generalized Pareto type II, Rayleigh generalized Pareto type II and Exponential generalized Pareto type II distributions), Yousof et al. [66] (Topp Leone Poisson Pareto type II distribution), Goual and Yousof [43] (Pareto type II inverse Rayleigh), Gad et al. [27] (Burr type XII Pareto type II, Pareto type II Burr type XII and Pareto type II Pareto type II distributions), Yadav et al. [65] (Topp Leone Pareto type II distribution) and Ibrahim and Yousof [36] (Poisson Burr X generalized 


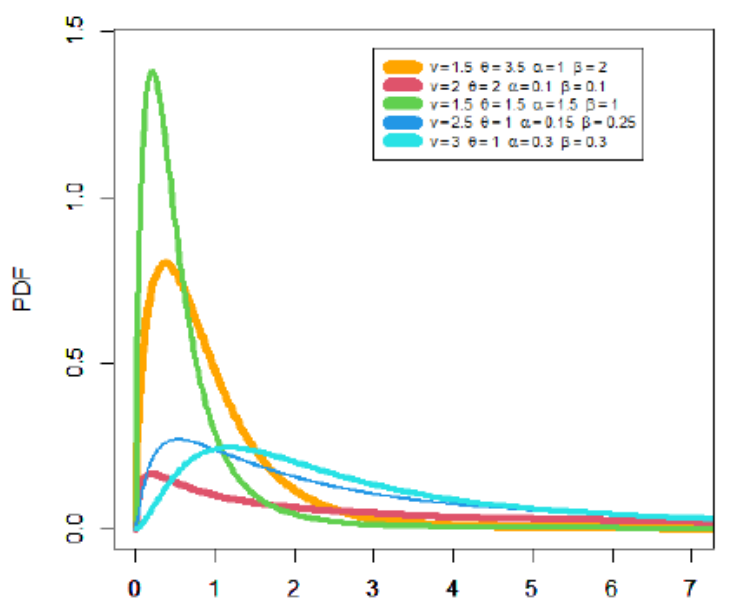

(a)

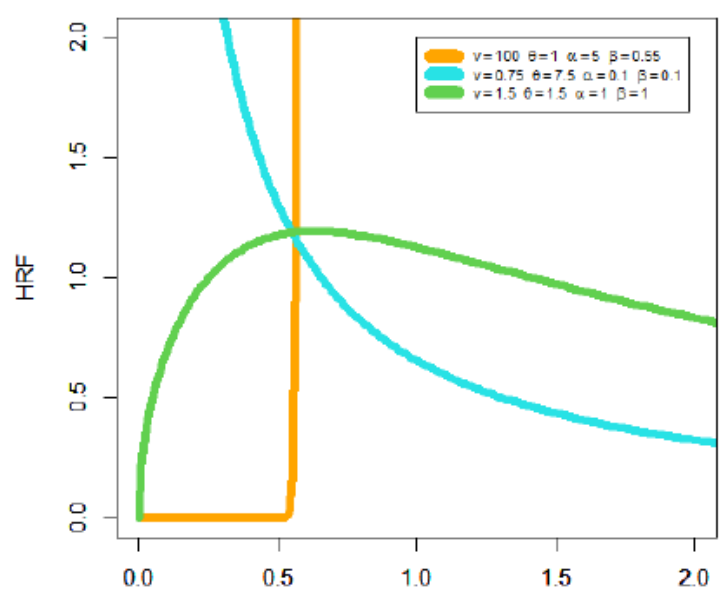

(b)

Figure 1. PDF and HRF plots for some selected parameters value.

Pareto type II and Poisson Rayleigh generalized Pareto type II distributions). To illustrate the flexibility of the new PDF and its corresponding HRF we present Figure 1. Figure 1(a) gives some PDF plots for some selected parameters value. Figure 1(b) gives some HRF plots for some selected parameters value. Based on Figure 1(a) the OBP density can be right skewed with heavy tail shape. Based on Figure 1(b) the OBP HRF can be "Jshape" ( $v=100, \theta=1, \alpha=5, \beta=0.55)$, "decreasing" $(v=0.75, \theta=7.5, \alpha=0.1, \beta=0.1)$ and "upside down (or increasing-constant-decreasing)" $(v=1.5=1.5, \alpha=\beta=1$ ).

\section{Extensions via copula}

In this section, we derive some new bivariate type OBP (BOBP) models using Farlie Gumbel Morgenstern (FGM) copula (see Kotz and Johnson [50], Kotz and Johnson [51], Gumbel [44] and Gumbel [45]), modified FGM copula (see Rodriguez-Lallena and Ubeda-Flores [60]) and Clayton copula and Renyi entropy copula (Pougaza and Djafari [59]). The multivariate OBP (MOBP) type is also presented. However, future works may be allocated to study these new models. First, we consider the joint CDF of the FGM family, where $\mathbf{H}_{\rho}(\tau, \varsigma)=\left.\tau \varsigma(1+\rho \bar{\tau} \bar{\varsigma})\right|_{\bar{\tau}=1-\tau, \bar{\varsigma}=1-\varsigma}$ with the marginal functions $\tau=F_{\xi_{1}}\left(\mathbf{z}_{1}\right), \varsigma=F_{\xi_{2}}\left(\mathbf{z}_{2}\right), \rho \in(-1,1)$ is a dependence parameter and for every $\tau, \varsigma \in(0,1), \mathbf{H}(\tau, 0)=\mathbf{H}(0, \varsigma)=0$ which is "grounded minimum" and $\mathbf{H}(\tau, 1)=\tau$ and $\mathbf{H}(1, \varsigma)=\varsigma$ which is "grounded maximum", $\mathbf{H}\left(\tau_{1}, \varsigma_{1}\right)+\mathbf{H}\left(\tau_{2}, \varsigma_{2}\right)-\mathbf{H}\left(\tau_{1}, \varsigma_{2}\right)-\mathbf{H}\left(\tau_{2}, \varsigma_{1}\right) \geq 0$. For more details see Elgohari and Yousof [25], Elgohari and Yousof [26], Al-babtain et al. [3], Ali et al. [4] and Ali et al. [5].

\subsection{BOBP type via FGM copula}

A copula is continuous in $\tau$ and $\varsigma$; actually, it satisfies the stronger Lipschitz condition, where

$$
\left|\mathbf{H}\left(\tau_{2}, \varsigma_{2}\right)-\mathbf{H}\left(\tau_{1}, \varsigma_{1}\right)\right| \leq\left|\tau_{2}-\tau_{1}\right|+\left|\varsigma_{2}-\varsigma_{1}\right| .
$$

For $0 \leq \tau_{1} \leq \tau_{2} \leq 1$ and $0 \leq \varsigma_{1} \leq \varsigma_{2} \leq 1$, we have

$$
\operatorname{Pr}\left(\tau_{1} \leq \tau \leq \tau_{2}, \varsigma_{1} \leq \varsigma \leq \varsigma_{2}\right)=\mathbf{H}\left(\tau_{1}, \varsigma_{1}\right)+\mathbf{H}\left(\tau_{2}, \varsigma_{2}\right)-\mathbf{H}\left(\tau_{1}, \varsigma_{2}\right)-\mathbf{H}\left(\tau_{2}, \varsigma_{1}\right) \geq 0 .
$$


Then, setting $\bar{\tau}=1-\left.F_{\underline{\xi}_{1}}\left(\mathbf{z}_{1}\right)\right|_{[\bar{\tau}=(1-\tau) \in(0,1)]}$ and $\bar{\varsigma}=1-\left.F_{\underline{\xi}_{2}}\left(\mathbf{z}_{2}\right)\right|_{[\varsigma=(1-\varsigma) \in(0,1)]}$, we can esaily obtain the joint CDF of the FGM family. The joint PDF can then be derived from $c_{\rho}(\tau, \varsigma)=1+\left.\rho \tau^{\circ} \varsigma^{\circ}\right|_{\left(\tau^{\prime}=1-2 \tau \text { and } \varsigma^{\prime}=1-2 \varsigma\right)}$ or from $c\left(\mathbf{z}_{1}, \mathbf{z}_{2}\right)=\mathbf{H}\left(F_{\underline{\xi}_{1}}\left(\mathbf{z}_{1}\right), F_{\underline{\xi}_{2}}\left(\mathbf{z}_{2}\right)\right) f_{\underline{\xi}_{1}}\left(\mathbf{z}_{1}\right) f_{\underline{\xi}_{2}}\left(\mathbf{z}_{2}\right)$.

\subsection{BOBP type via modified FGM copula}

The modified FGM copula is defined as $\mathbf{H}_{\rho}(\tau, \varsigma)=\left.\tau \varsigma[1+\rho \vartheta(\tau) \boldsymbol{\Phi}(\varsigma)]\right|_{\rho \in(-1,1)}$ or $\mathbf{H}_{\rho}(\tau, \varsigma)=\tau \varsigma+$ $\left.\rho \widetilde{\vartheta}_{\tau} \widetilde{\boldsymbol{\Phi}}_{\varsigma}\right|_{\rho \in(-1,1)}$, where $\widetilde{\vartheta}_{\tau}=\tau \vartheta(\tau)$, and $\widetilde{\boldsymbol{\Phi}}_{\varsigma}=\varsigma \mathbf{\Phi}(\varsigma)$ and $\vartheta(\tau)$ and $\boldsymbol{\Phi}(\varsigma)$ are two continuous functions on $(0,1)$ with $\vartheta(0)=\vartheta(1)=\boldsymbol{\Phi}(0)=\boldsymbol{\Phi}(1)=0$. Let

$$
\begin{aligned}
& \alpha_{1}(\tau)=\inf \left\{\widetilde{\vartheta}_{\tau}:\left.\frac{\partial}{\partial \tau} \widetilde{\vartheta}_{\tau}\right|_{\sigma_{1}}\right\}<0, \alpha_{2}(\tau)=\sup \left\{\widetilde{\vartheta}_{\tau}:\left.\frac{\partial}{\partial \tau} \widetilde{\vartheta}_{\tau}\right|_{\sigma_{1}(\varsigma)}\right\}<0, \\
& \theta_{1}(\varsigma)=\inf \left\{\widetilde{\boldsymbol{\Phi}}_{\varsigma}:\left.\frac{\partial}{\partial \varsigma} \widetilde{\boldsymbol{\Phi}}_{\varsigma}\right|_{\sigma_{2}}\right\}>0, \theta_{2}(\varsigma)=\sup \left\{\widetilde{\boldsymbol{\Phi}}_{\varsigma}:\left.\frac{\partial}{\partial \varsigma} \widetilde{\boldsymbol{\Phi}}_{\varsigma}\right|_{\sigma_{2}(\varsigma)}\right\}>0 .
\end{aligned}
$$

Then, $\quad 1 \leq \min \left(\alpha_{1}(\tau) \alpha_{2}(\tau), \theta_{1}(\varsigma) \theta_{2}(\varsigma)\right)<\infty, \quad$ where $\quad \tau \frac{\partial}{\partial \tau} \vartheta(\tau)=\frac{\partial}{\partial \tau} \widetilde{\vartheta}_{\tau}-\vartheta(\tau), \quad \sigma_{1}(\varsigma)=$ $\left\{\tau: \tau \in(0,1) \mid \frac{\partial}{\partial \tau} \widetilde{\vartheta}_{\tau}\right.$ exists $\}$ and $\sigma_{2}(\varsigma)=\left\{\varsigma: \varsigma \in(0,1) \mid \frac{\partial}{\partial \varsigma} \widetilde{\boldsymbol{\Phi}}_{\varsigma}\right.$ exists $\}$.

- Type-I

Consider the following functional form for both $\widetilde{\vartheta}_{\tau}$ and $\widetilde{\boldsymbol{\Phi}}_{\varsigma}$ where $\widetilde{\vartheta}_{\tau}=\tau\left[1-F_{\underline{\xi}_{1}}(\tau)\right]$ and $\widetilde{\boldsymbol{\Phi}}_{\varsigma}=$ $\varsigma\left[1-F_{\underline{\xi}_{2}}(\varsigma)\right]$. Then, the BOBP-FGM (Type-I) can be derived from $\mathbf{H}_{\rho}(\tau, \varsigma)=\tau \varsigma+\left.\rho \widetilde{\vartheta}_{\tau} \widetilde{\mathbf{\Phi}}_{\varsigma}\right|_{\rho \in(-1,1)}$

- Type-II

Let $\vartheta(\tau)^{\circ}$ and $\boldsymbol{\Phi}(\varsigma)^{\cdot}$ be two functional forms satisfy all the conditions stated earlier where $\left.\vartheta(\tau)^{\cdot}\right|_{\left(\rho_{1}>0\right)}=$ $\tau^{\rho_{1}}(1-\tau)^{1-\rho_{1}}$ and $\left.\boldsymbol{\Phi}(\varsigma)^{\cdot}\right|_{\left(\rho_{2}>0\right)}=\varsigma^{\rho_{2}}(1-\varsigma)^{1-\rho_{2}}$. Then, the corresponding BOBP-FGM (Type-II) can be derived from $\mathbf{H}_{\rho, \rho_{1}, \rho_{2}}(\tau, \varsigma)=\tau \varsigma\left[1+\rho \vartheta(\tau)^{\cdot} \mathbf{\Phi}(\varsigma)^{\cdot}\right]$.

- Type-III

Let $\widetilde{\vartheta(\tau)}=\left.\tau[\log (1+\bar{\tau})]\right|_{\bar{\tau}=1-\tau}$ and $\widetilde{\boldsymbol{\Phi}(\varsigma)}=\left.\varsigma[\log (1+\varsigma)]\right|_{\varsigma=1-\varsigma}$. In this case, one can also derive a closed form expression for the associated CDF of the BOBP-FGM (Type-III) from $\mathbf{H}_{\rho}(\tau, \varsigma)=\tau \varsigma(1+\rho \widetilde{\vartheta(\tau)} \widetilde{\boldsymbol{\Phi}(\varsigma)})$.

- Type-IV

The CDF of the BOBP-FGM (Type-IV) model can be derived from $\mathbf{H}(\tau, \varsigma)=\tau F_{\underline{\xi}_{2}}^{-1}(\varsigma)+\varsigma F_{\underline{\xi}_{1}}^{-1}(\tau)-$ $F_{\underline{\xi}_{1}}^{-1}(\tau) F_{\underline{\xi}_{2}}^{-1}(\varsigma)$ where $F_{\underline{\xi}_{1}}^{-1}(\tau)$ and $F_{\underline{\xi}_{2}}^{-1}(\varsigma)$ can be easily derived from (5).

\subsection{BOBP type via Ali-Mikhail-Haq copula}

Under the stronger Lipschitz condition, the joint CDF of the archimedean Ali-Mikhail-Haq copula can expressed as

$$
\mathbf{H}\left(\varsigma_{1}, \varsigma_{2}\right)=\left.\frac{\varsigma_{1} \varsigma_{2}}{1-\rho \overline{\varsigma_{1} \varsigma_{2}}}\right|_{\rho \in(-1,1)}
$$

the corresponding joint PDF of the archimedean Ali-Mikhail-Haq copula can expressed as

$$
\mathbf{h}\left(\varsigma_{1}, \varsigma_{2}\right)=\left.\frac{1-\rho+2 \rho \frac{\varsigma_{1} \varsigma_{2}}{1-\rho \overline{1}_{1} \varsigma_{2}}}{\left[1-\rho \overline{\varsigma_{1} \varsigma_{2}}\right]^{2}}\right|_{\rho \in(-1,1)},
$$

sitting $\overline{\varsigma_{1}}=1-F_{\underline{\xi}_{1}}\left(\mathbf{z}_{1}\right)$ and $\overline{\varsigma_{2}}=1-F_{\underline{\xi}_{1}}\left(\mathbf{z}_{1}\right)$ we can derive the joint CDF and the joint PDF of the BOBP type via Ali-Mikhail-Haq copula. 


\subsection{BOBP and MOBP type via Clayton copula}

The Clayton copula can be considered as $\mathbf{H}\left(\varsigma_{1}, \varsigma_{2}\right)=\left.\left[\left(1 / \varsigma_{1}\right)^{\rho}+\left(1 / \varsigma_{2}\right)^{\rho}-1\right]^{-\rho^{-1}}\right|_{\rho \in(0, \infty)}$. Setting $\varsigma_{1}=F_{\boldsymbol{\Phi}_{1}}(t)$ and $\varsigma_{2}=F_{\boldsymbol{\Phi}_{2}}(x)$, the BOBP type can be derived from $\mathbf{H}\left(\varsigma_{1}, \varsigma_{2}\right)=\mathbf{H}\left(F_{\underline{\xi}_{1}}(t), F_{\boldsymbol{\Phi}_{2}}(x)\right)$. Similarly, the MOBP ( $m$-dimensional extension) from the above can be derived from $\mathbf{H}\left(\varsigma_{n}\right)=\left(\sum_{n=1}^{m} \varsigma_{n}^{-\rho}+1-m\right)^{-\rho^{-1}}$.

\subsection{BOBP type via Renyi's entropy copula}

Using the theorem of Pougaza and Djafari [59] where $\mathbf{H}(\tau, \varsigma)=\mathbf{z}_{2} \tau+\mathbf{z}_{1} \varsigma-\mathbf{z}_{1} \mathbf{z}_{2}$, the associated BOBP will be $\mathbf{H}(u, \varsigma)=\mathbf{H}\left(F_{\underline{\mathbf{V}}_{1}}\left(\mathbf{z}_{1}\right), F_{\underline{\mathbf{V}}_{2}}\left(\mathbf{z}_{2}\right)\right)$.

\section{Mathematical properties}

\subsection{Asymptotics and quantile function}

In mathematical analysis, the asymptotic analysis is used for describing the limiting behavior of some functions. Asymptotics derivations for the CDF, PDF and HRF can be obtained for the new model. The asymptotics of the $\mathrm{CDF}, \mathrm{PDF}$ and $\mathrm{HRF}$ as $\mathrm{z} \rightarrow 0$ are given by

$$
\left.F_{\underline{\xi}}(z) \sim \theta\left[1-\nabla_{\beta}(z)^{-\alpha}\right]^{v}\right|_{z \longrightarrow 0},\left.f_{\underline{\xi}}(z) \sim v \theta \frac{\alpha}{\beta} \nabla_{\beta}(z)^{-(\alpha+1)}\left[1-\nabla_{\beta}(z)^{-\alpha}\right]^{v-1}\right|_{z \rightarrow 0},
$$

and

$$
\left.h_{\underline{\xi}}(z) \sim v \theta \frac{\alpha}{\beta} \nabla_{\beta}(z)^{-(\alpha+1)}\left[1-\nabla_{\beta}(z)^{-\alpha}\right]^{v-1}\right|_{z \longrightarrow 0},
$$

The asymptotics of CDF, PDF and $\mathrm{HRF}$ as $\mathrm{z} \rightarrow \infty$ are given by

$$
\left.S_{\underline{\xi}}(z) \sim v^{\theta} \nabla_{\beta}(z)^{-\alpha \theta}\right|_{z \longrightarrow \infty},\left.f_{\underline{\xi}}(z) \sim \theta v^{\theta} \frac{\alpha}{\beta} \nabla_{\beta}(z)^{-\alpha v-1}\right|_{z \longrightarrow \infty}
$$

and

$$
\left.h_{\underline{\xi}}(z) \sim \theta \frac{\alpha}{\beta} \nabla_{\beta}(z)^{-1}\right|_{z \longrightarrow \infty},
$$

For simulation of this new model, we obtain the quantile function (QF) of $Z$ (by inverting (5)), say $\mathbf{z}_{u}=F^{-1}(u)$, as

$$
\mathbf{z}_{u}=\beta\left\{\left[1-\frac{\left(1-u^{\frac{1}{\theta}}\right)^{\frac{1}{v}}}{u^{\frac{1}{v \theta}}+\left(1-u^{\frac{1}{\theta}}\right)^{\frac{1}{v}}}\right]^{-\frac{1}{\alpha}}-1\right\} .
$$

Equation (7) is used for simulating the new model.

\subsection{Useful representations}

Due to Alizadeh et al. (2017a), the PDF in (6) can be expressed as

$$
f(\mathbf{z})=\sum_{s=0}^{\infty} \eta_{s} g_{s} \cdot \alpha,\left.\beta(\mathbf{z})\right|_{s^{\bullet}=1+s}
$$

where

$$
\eta_{s}=\frac{v \theta}{s^{\bullet}} \sum_{j_{1}, j_{2}=0}^{\infty} \sum_{j_{3}=s}^{\infty}(-1)^{j_{2}+k+s}\left(\begin{array}{c}
-(1+\theta) \\
j_{1}
\end{array}\right)\left(\begin{array}{c}
-\left[v\left(1+j_{1}\right)+1\right] \\
j_{2}
\end{array}\right)\left(\begin{array}{c}
v\left(1+j_{1}\right)+j_{2}+1 \\
j_{3}
\end{array}\right)\left(\begin{array}{c}
j_{3} \\
s
\end{array}\right),
$$




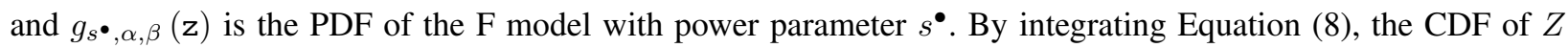
becomes

$$
F(\mathbf{z})=\sum_{s=0}^{\infty} \eta_{s} G_{s} \cdot \alpha, \beta(\mathbf{z}),
$$

where $G_{s^{\bullet}, \alpha, \beta}(\mathbf{z})$ is the CDF of the Pareto type II distribution with power parameter $s^{\bullet}$.

\subsection{Moments and incomplete moments}

The $\mathbf{r}^{\text {th }}$ ordinary moment of $Z$ is given by

$$
\mu_{\mathbf{r}, z}^{\prime}=\mathbf{E}\left(\mathbf{z}^{\mathbf{r}}\right)=\int_{-\infty}^{\infty} \mathbf{z}^{\mathbf{r}} f(\mathbf{z}) d \mathbf{z}
$$

then we obtain

$$
\mu_{\mathbf{r}, Z}^{\prime}=\left.\sum_{s=0}^{\infty} \sum_{\kappa=0}^{\mathbf{r}} \eta_{s} s^{\bullet} \beta^{\mathbf{r}}(-1)^{\kappa}\left(\begin{array}{l}
\mathbf{r} \\
\kappa
\end{array}\right) \mathcal{B}\left(s^{\bullet}, 1+\frac{1}{\alpha}(\kappa-\mathbf{r})\right)\right|_{(\alpha>\mathbf{r})},
$$

where $\mathcal{B}\left(w_{1}, w_{2}\right)=\int_{0}^{1} m^{w_{1}-1}(1-m)^{w_{2}-1} d m$. Setting $\mathbf{r}=1,2,3$ and 4 in (10), we have

$$
\begin{aligned}
\mathbf{E}(\mathrm{Z}) & =\left.\sum_{s=0}^{\infty} \sum_{\kappa=0}^{1} \eta_{s} s^{\bullet} \beta(-1)^{\kappa}\left(\begin{array}{l}
1 \\
\kappa
\end{array}\right) \mathcal{B}\left(s^{\bullet}, \frac{1}{\alpha}(\kappa-1)+1\right)\right|_{(\alpha>1)}, \\
\mathbf{E}\left(\mathrm{Z}^{2}\right) & =\left.\sum_{s=0}^{\infty} \sum_{\kappa=0}^{2} \eta_{s} s^{\bullet} \beta^{2}(-1)^{\kappa}\left(\begin{array}{l}
2 \\
\kappa
\end{array}\right) \mathcal{B}\left(s^{\bullet}, \frac{1}{\alpha}(\kappa-2)+1\right)\right|_{(\alpha>2)}, \\
\mathbf{E}\left(\mathrm{Z}^{3}\right) & =\left.\sum_{s=0}^{\infty} \sum_{\kappa=0}^{3} \eta_{s} s^{\bullet} \beta^{3}(-1)^{\kappa}\left(\begin{array}{l}
3 \\
\kappa
\end{array}\right) \mathcal{B}\left(s^{\bullet}, \frac{1}{\alpha}(\kappa-3)+1\right)\right|_{(\alpha>3)},
\end{aligned}
$$

and

$$
\mathbf{E}\left(\mathrm{Z}^{4}\right)=\left.\sum_{s=0}^{\infty} \sum_{\kappa=0}^{3} \eta_{s} s^{\bullet} \beta^{4}(-1)^{\kappa}\left(\begin{array}{l}
4 \\
\kappa
\end{array}\right) \mathcal{B}\left(s, \frac{1}{\alpha}(\kappa-4)+1\right)\right|_{(\alpha>4)},
$$

where $\mathbf{E}(\mathrm{Z})=\mu_{1}^{\prime}$ is the mean of $Z$. The $\mathbf{r}^{t h}$ incomplete moment, say $\mathcal{I}_{\mathbf{r}}(t)$, of $Z$ can be expressed, from (9), as

$$
\mathcal{I}_{\mathbf{r}, \mathbf{Z}}(t)=\int_{-\infty}^{t} \mathbf{z}^{\mathbf{r}} f(\mathbf{z}) d \mathbf{z}=\sum_{s=0}^{\infty} \eta_{s} \int_{-\infty}^{t} \mathbf{z}^{\mathbf{r}} g_{s} \cdot, \alpha, \beta(\mathbf{z}) d \mathbf{z}
$$

then

$$
\mathcal{I}_{\mathbf{r}, \mathrm{Z}}(t)=\left.\sum_{s=0}^{\infty} \sum_{\kappa=0}^{\mathbf{r}} \eta_{s} s^{\bullet} \beta^{\mathbf{r}}(-1)^{\kappa}\left(\begin{array}{l}
\mathbf{r} \\
\kappa
\end{array}\right) \mathcal{B}_{t}\left(s^{\bullet}, 1+\frac{1}{\alpha}(\kappa-\mathbf{r})\right)\right|_{(\alpha>\mathbf{r})},
$$

where $\mathcal{B}_{y}\left(w_{1}, w_{2}\right)=\int_{0}^{y} m^{w_{1}-1}(1-m)^{w_{2}-1} d m$. The first incomplete moment given by (11) with $\mathbf{r}=1$ as

$$
\mathcal{I}_{1, \mathrm{Z}}(t)=\left.\sum_{s=0}^{\infty} \sum_{\kappa=0}^{1} \eta_{s} s^{\bullet} \beta(-1)^{\kappa}\left(\begin{array}{l}
1 \\
\kappa
\end{array}\right) \mathcal{B}_{t}\left(s \bullet, \frac{1}{\alpha}(\kappa-1)+1\right)\right|_{(\alpha>1)} .
$$

The index of dispersion IxDis or the variance to mean ratio can derived as $\operatorname{IxDis}(\mathbf{z})=\mu_{2} / \mu_{1}^{\prime}$. It is a measure used to quantify whether a set of observed occurrences are clustered or dispersed compared to a standard statistical model. 


\subsection{Numerical ans graphical analysis}

By analyzing the mean $[\mathbf{E}(\mathrm{Z})]$, variance $[\operatorname{Var}(\mathrm{Z})]$, skewness $[\operatorname{Skew}(\mathrm{Z})]$, kurtosis $[\operatorname{Kur}(\mathrm{Z})]$ and $\operatorname{IxDis}(\mathrm{Z})$ numerical in Table 1 we noted that, the $\operatorname{Skew}(Z)$ of the OBP distribution can range in the interval $(-69216.87,98983.16)$. The spread for the $\operatorname{Kur}(\mathrm{Z})$ of the OBP model is ranging from 74.17124 to $\infty$. The IxDis $(\mathrm{Z})$ for the OBP model can be in $(0,1)$ an dalso $>1$ so it may be used as an "under-dispersed" and "over-dispersed" model. Figure 2 gives some three dimensional skewness plots for parameter $\alpha$. The flexibility of the skewness of the new model are proved in Figures 2 and 3 using some some three dimensional plots. The flexibility of the kurtosis of the new model are proved in Figures 4 and 5 using some some three dimensional plots. Figure 3 gives some three dimensional skewness plots for parameter $\theta$. Figure 4 gives some three dimensional kurtosis plots for parameter $\alpha$. Finally, Figure 5 gives three dimensional kurtosis plots for parameter $\theta$.

Table 1: E(Z), $\operatorname{Var}(\mathrm{Z}), \operatorname{Skew}(\mathrm{Z})$ and $\operatorname{Kur}(\mathrm{Z})$ of the OBP model.

\begin{tabular}{ccccccccc}
\hline \hline$v$ & $\theta$ & $\alpha$ & $\beta$ & $\mathbf{E}(\mathrm{Z})$ & $\operatorname{Var}(\mathrm{Z})$ & $\operatorname{Skew}(\mathrm{Z})$ & $\operatorname{Kur}(\mathrm{Z})$ & $\operatorname{IxDis}(\mathrm{Z})$ \\
\hline \hline 1 & 1.5 & 10 & 1.5 & 0.1071429 & 0.01324568 & 2.4825320 & 13.63030 & 0.1236264 \\
5 & & & & 0.1003165 & 0.0005641241 & 0.4022094 & 4.077114 & 0.0056234 \\
10 & & & & 0.1033713 & 0.0001518643 & 0.0196449 & 3.829237 & 0.0014691 \\
50 & & & & 0.1066996 & $6.535887 \mathrm{e}-06$ & $-69216.87^{*}$ & 8673587 & $6.126 \times 10^{-5}$ \\
100 & & & & $7.9602 \times 10^{-05}$ & $9.18712 \times 10^{-07}$ & 120.4033 & 14497.96 & 0.1154139 \\
200 & & & & $1.1781 \times 10^{-11}$ & $1.35973 \times 10^{-12}$ & $98983.16^{*}$ & $\infty *$ & 0.1154218 \\
& & & & & & & & \\
5 & 0.5 & 5 & 5 & 0.9647359 & 0.16261690 & 2.478782 & 16.67835 & 0.168561 \\
& 1 & & & 0.7659348 & 0.04623618 & 1.026232 & 6.366972 & 0.060366 \\
& 10 & & & 0.4800206 & 0.00957576 & -0.3233682 & 3.074851 & 0.019949 \\
& 50 & & & 0.3618845 & 0.00549617 & -0.3826962 & 3.042526 & 0.015188 \\
& 100 & & & 0.3201573 & 0.00438074 & -0.3796808 & 3.025701 & 0.013683 \\
& 200 & & & 0.2828904 & 0.00348778 & -0.3717237 & 3.008953 & 0.012329 \\
& & & & & & & & \\
2.5 & 2.5 & 5 & 10 & 1.076590 & 0.22120710 & 0.729365 & 4.209933 & 0.2054701 \\
& 50 & & 0.101967 & 0.00179817 & 0.587341 & 3.757355 & 0.0176349 \\
& 100 & & 0.050832 & 0.00044447 & 0.579787 & 4.74781 & 0.0087438 \\
& 200 & & 0.025378 & 0.00011049 & 3.266505 & -14.47678 & 0.0043536 \\
& 500 & & 0.010142 & $1.7618 \times 10^{-5}$ & 10.98053 & $-74.17124 *$ & 0.0017371 \\
& & & & & & & & \\
1.5 & 3.5 & 1.5 & 5 & 0.119954 & 0.006565 & 1.215589 & 5.318137 & 0.054729 \\
& & 20 & 0.479814 & 0.105038 & 1.215591 & 5.318139 & 0.218914 \\
& & 50 & 1.199535 & 0.656487 & 1.215591 & 5.318139 & 0.547285 \\
& & 200 & 4.798140 & 10.50380 & 1.215591 & 5.318139 & 2.189139 \\
& & 500 & 11.99535 & 65.64873 & 1.215591 & 5.318139 & 5.472848 \\
& & 1000 & 23.99070 & 262.5949 & 1.215591 & 5.318139 & 10.94570 \\
\hline \hline
\end{tabular}

\subsection{Some generating functions $(G F)$}

The moment generating function (MGF) can be derived using (8) as

$$
M_{\mathrm{Z}}(t)=\sum_{s=0}^{\infty} \eta_{s} M_{s} \bullet(t ; \beta),
$$



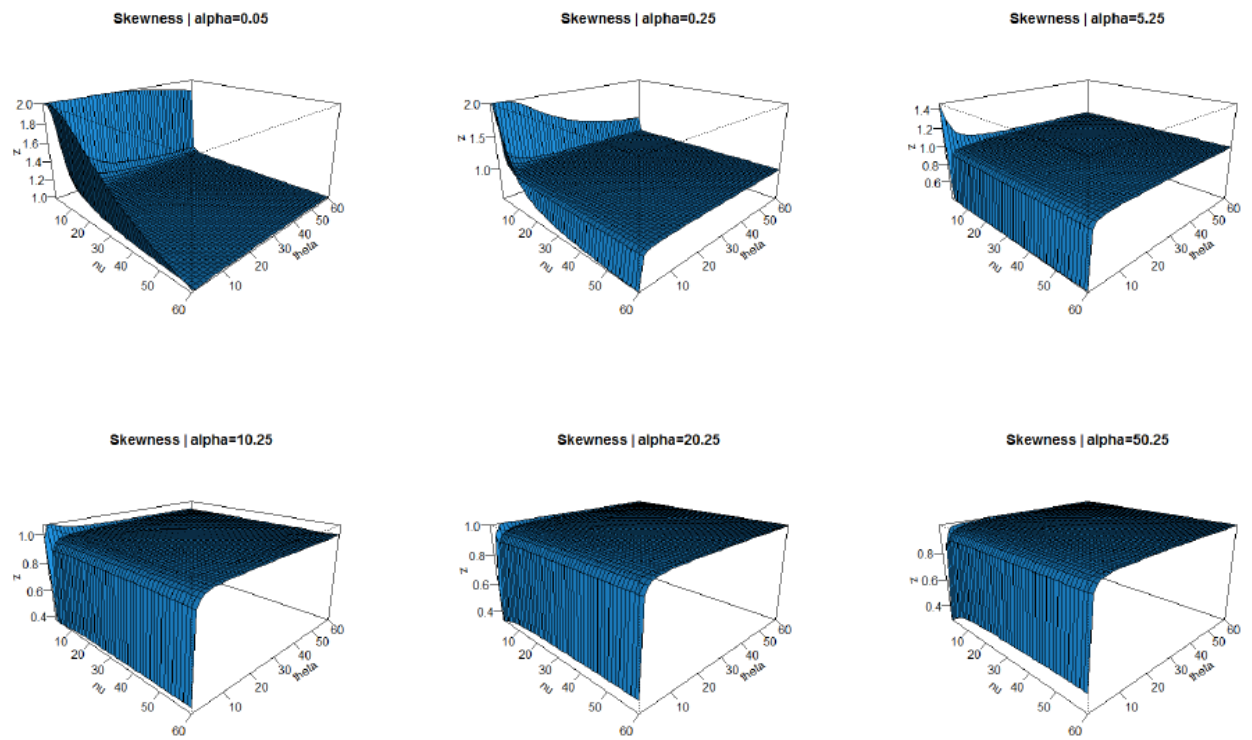

Figure 2. Three dimensional Skewness plots for parameter $\alpha$.
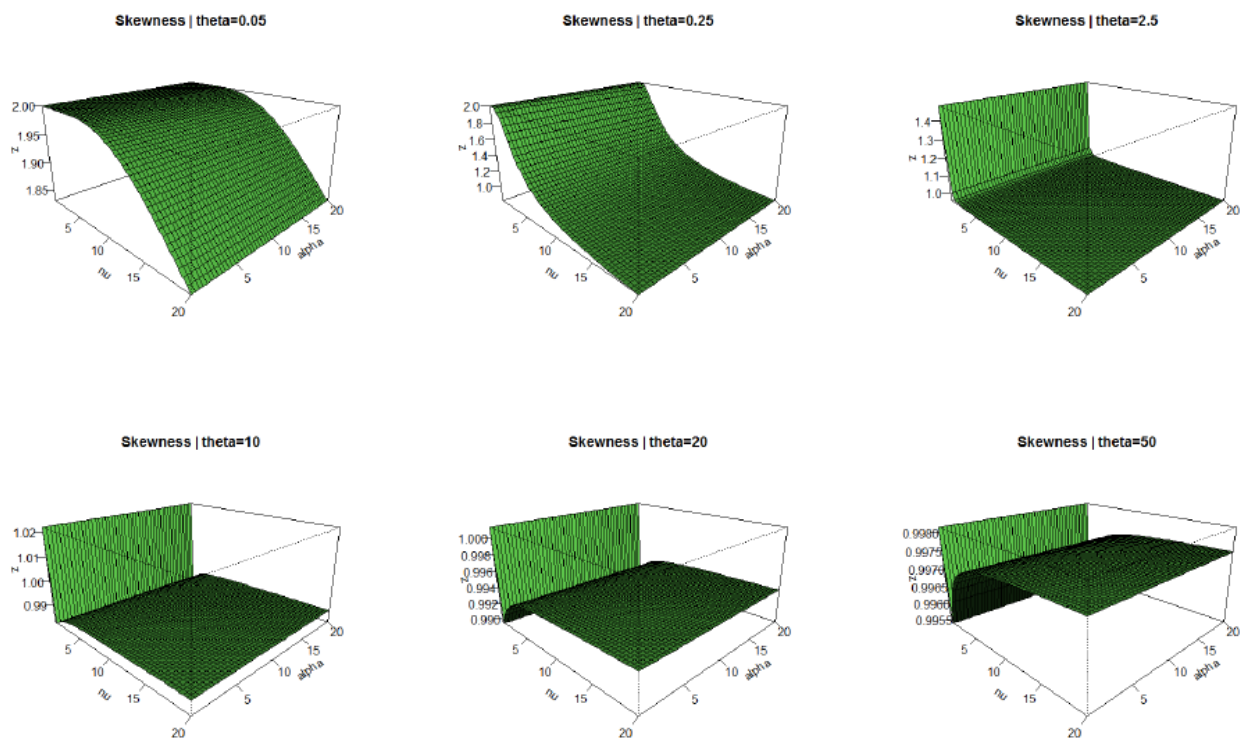

Figure 3. Three dimensional Skewness plots for parameter $\theta$.

where $M_{s} \bullet(t ; \alpha, \beta)$ is the MGF of the ExpP model, then

$$
M_{\mathrm{Z}}(t)=\left.\sum_{s=0}^{\infty} \sum_{\mathbf{r}=0}^{\infty} \sum_{\kappa=0}^{\mathbf{r}} \frac{t^{\mathbf{r}}}{\mathbf{r} !} \eta_{s} s^{\bullet} \beta^{\mathbf{r}}(-1)^{\kappa}\left(\begin{array}{l}
\mathbf{r} \\
\kappa
\end{array}\right) \mathcal{B}\left(s^{\bullet}, 1+\frac{1}{\alpha}(\kappa-\mathbf{r})\right)\right|_{(\alpha>\mathbf{r})} .
$$



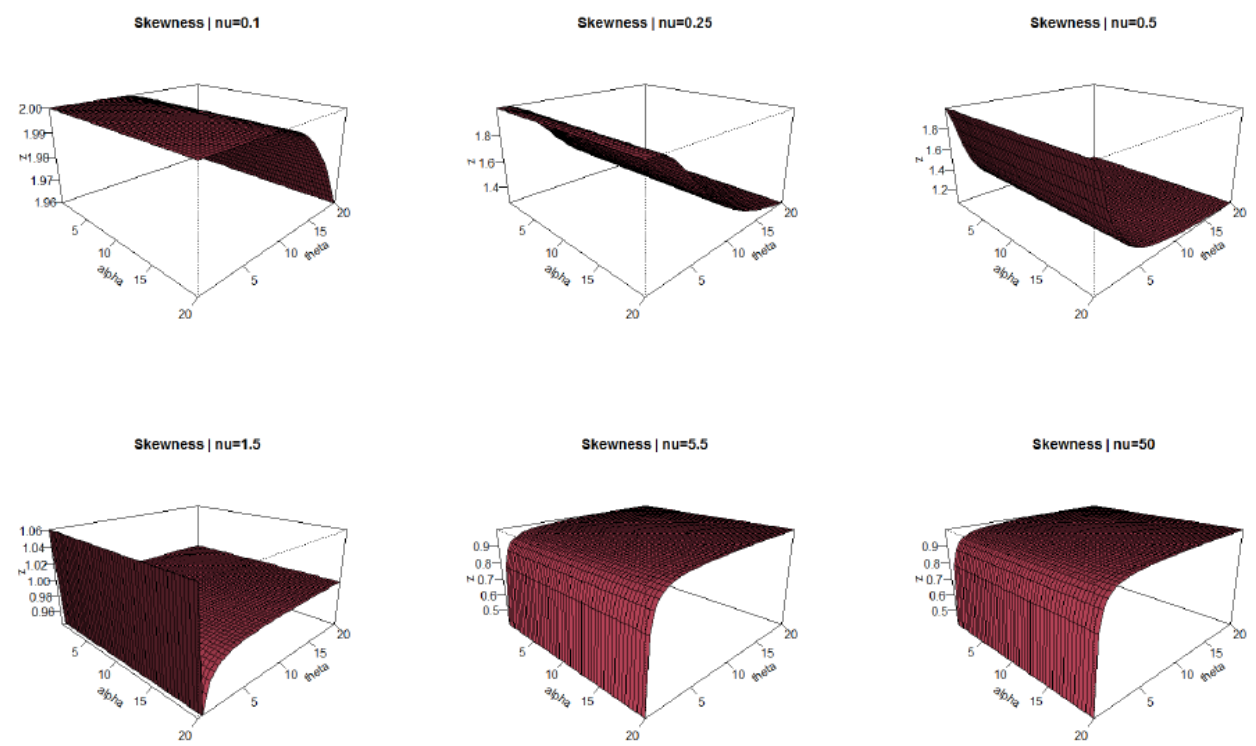

Figure 4. Three dimensional Skewness plots for parameter v.
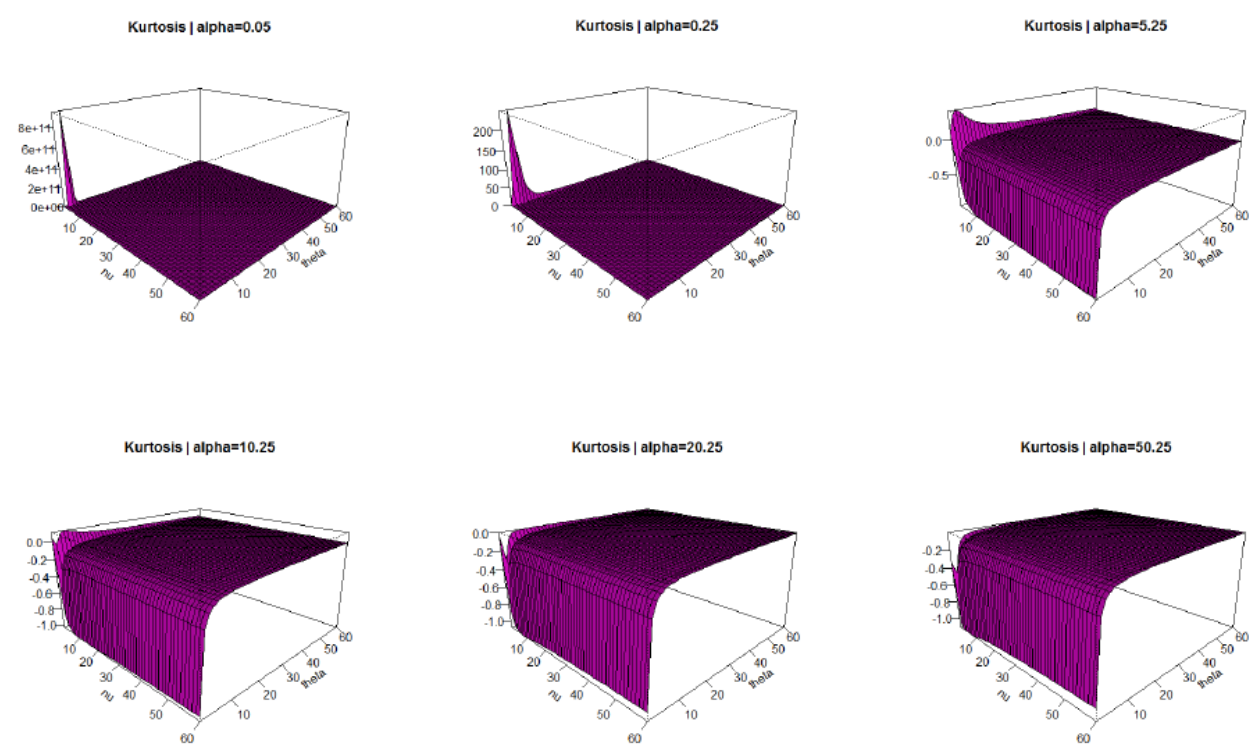

Figure 5. Three dimensional kurtosis plots for parameter $\alpha$.

The first $\mathbf{r}$ derivatives of $M_{\mathrm{Z}}(t)$, with respect to $t$ at $t=0$, yield the first $\mathbf{r}$ moments about the origin, i.e., 

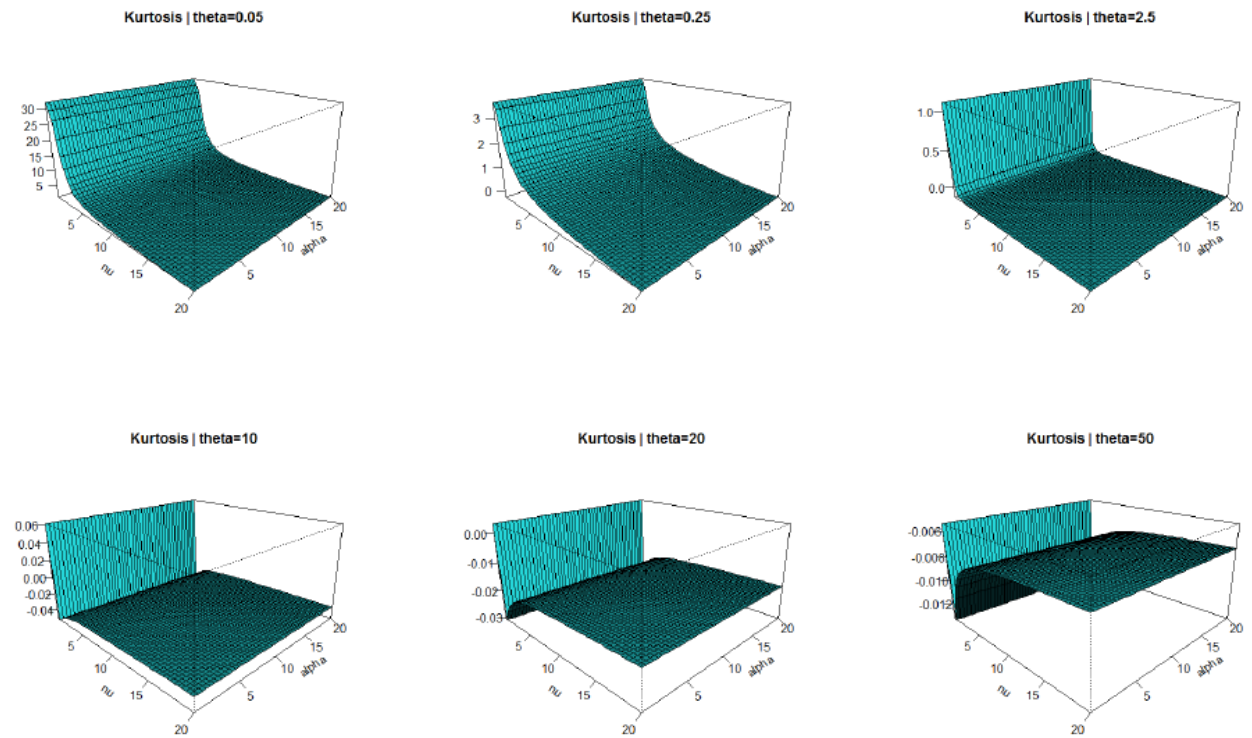

Figure 6. Three dimensional kurtosis plots for parameter $\theta$.
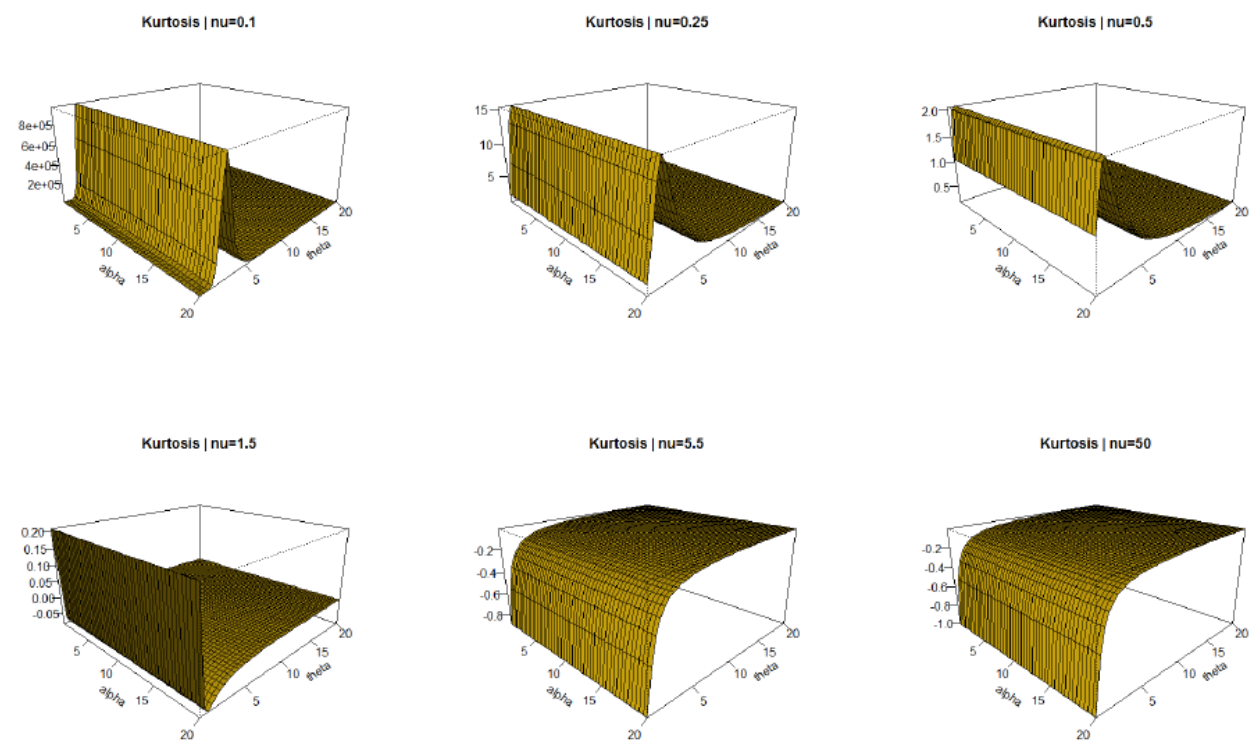

Figure 7. Three dimensional kurtosis plots for parameter v.

$$
\mu_{\mathbf{r}, \mathbf{z}}^{\prime}=\mathbb{E}\left(\mathbf{z}^{r}\right)=\left.\frac{d^{\mathbf{r}}}{d t^{\mathbf{r}}} M_{\mathbf{z}}(t)\right|_{(t=0 \text { and } \mathbf{r}=1,2,3, \ldots)},
$$


The generating function GF (CGF) is the logarithm of the MGF. Thus, $\mathrm{r}^{\text {th }}$ cumulant, say $\kappa_{\mathbf{r}}$, can be obtained from

$$
\kappa_{\mathbf{r}, \mathrm{Z}}=\left.\frac{d^{\mathbf{r}}}{d t^{\mathbf{r}}} \log \left[\sum_{s=0}^{\infty} \sum_{\mathbf{r}=0}^{\infty} \sum_{\kappa=0}^{\mathbf{r}} \frac{t^{\mathbf{r}}}{\mathbf{r} !} \eta_{s} s^{\bullet} \beta^{\mathbf{r}}(-1)^{\kappa}\left(\begin{array}{l}
\mathbf{r} \\
\kappa
\end{array}\right) \mathcal{B}\left(s^{\bullet}, 1+\frac{1}{\alpha}(\kappa-\mathbf{r})\right)\right]\right|_{(t=0, \text { and } \mathbf{r}=1,2,3, \ldots)} .
$$

The $1^{\text {st }}$ cumulant is the mean $\left(\kappa_{1, \mathrm{z}}=\mu_{1}^{\prime}\right)$, the $2^{\text {nd }}$ cumulant is the variance, and the $3^{\text {rd }}$ cumulant is the same as the $3^{\text {rd }}$ central moment $\kappa_{3, \mathrm{z}}=\mu_{3, \mathrm{z}}$. But $4^{\text {th }}$ and higher-order cumulants are not equal to central moments, that being said $\kappa_{1, \mathrm{z}}=\mu_{1, \mathrm{z}}^{\prime}, \kappa_{2, \mathrm{z}}=\mu_{2, \mathrm{z}}^{\prime}-\mu_{1, \mathrm{z}}^{\prime 2}=\mu_{2, \mathrm{z}}$ and $\kappa_{3, \mathrm{z}}=\mu_{3, \mathrm{z}}^{\prime}-3 \mu_{2, \mathrm{z}}^{\prime} \mu_{1, \mathrm{z}}^{\prime}+2 \mu_{1, \mathrm{z}}^{\prime 3}=\mu_{3, \mathrm{z}}$. In some cases theoretical treatments of problems in terms of cumulants are simpler than those using moments. In particular, when two or more RVs are statistically independent, the $\mathbf{r}^{\text {th }}$ order cumulant of their sum is equal to the sum of their $\mathbf{r}^{\text {th }}$ order cumulants. Moreover, the cumulants can be also obtained from $\left.\kappa_{\mathbf{r}, \mathrm{Z}}\right|_{\mathbf{r} \geq 1}=\mu_{\mathbf{r}, \mathrm{Z}}^{\prime}-\sum_{m=0}^{\mathbf{r}-1}\left(\begin{array}{c}\mathbf{r}-1 \\ m-1\end{array}\right) \mu_{\mathbf{r}-m, \mathrm{Z}}^{\prime} \kappa_{m, \mathrm{Z}}$.

\subsection{Reversed residual life function}

The $n^{t h}$ moment of the reversed residual life, say $V_{n, \mathrm{Z}}(t)=\mathbf{E}\left[\left.(t-\mathbf{z})^{n}\right|_{\mathbf{z} \leq t, t>0 \text { and } n=1,2, \ldots}\right]$. Then, $V_{n, \mathrm{Z}}(t)$ can written as

$$
V_{n, Z}(t)=\frac{1}{F_{v, \theta, \underline{\zeta}}(\mathrm{t})} \int_{0}^{t}(t-\mathbf{z})^{n} d F_{v, \theta, \underline{\zeta}}(\mathbf{z}) .
$$

Then, the $n^{\text {th }}$ moment of the reversed residual life of $Z$ becomes

$$
V_{n, \mathrm{Z}}(t)=\left.\frac{1}{F_{v, \theta, \underline{\zeta}}(\mathrm{t})} \sum_{s=0}^{\infty} \sum_{\kappa=0}^{\mathbf{r}} \eta_{s}^{*} s^{\bullet} \beta^{\mathbf{r}}(-1)^{\kappa}\left(\begin{array}{l}
\mathbf{r} \\
\kappa
\end{array}\right) \mathcal{B}_{t}\left(s^{\bullet}, 1+\frac{1}{\alpha}(\kappa-\mathbf{r})\right)\right|_{(\alpha>\mathbf{r})},
$$

where

$$
\eta_{s}^{*}=\eta_{s} \sum_{h=0}^{n}(-1)^{h}\left(\begin{array}{l}
n \\
h
\end{array}\right) t^{n-h} .
$$

\section{Estimation}

In this Section, non-Bayesian and Bayesian estimation methods are considered. In first subsection, we will consider four non-Bayesian estimation methods such as the MLE, OLSE, WLSE and the KE methods. In the second subsection, the Bayesian estimation method under the squared error loss function (SELF).

\subsection{Non-Bayesian estimation methods}

4.1.1. The $M L E$ Let $\mathrm{z}_{1}, \mathrm{z}_{2}, \ldots, \mathrm{z}_{n}$ be a random sample from size $n$ from the OBP distribution with parameters $v, \theta, \alpha$ and $\beta$. Let $\underline{\xi}^{\top}$ be the $4 \times 1$ parameter vector. For determining the MLE of $\underline{\xi}$, we have the log-likelihood function

$$
\begin{aligned}
\ell(\underline{\xi})= & (v-1) \sum_{i=1}^{n} \log \left[1-\nabla_{\beta}\left(\mathbf{z}_{i}\right)^{-\alpha}\right]+n \log \left(v \theta \frac{\alpha}{\beta}\right)-(\alpha v \theta+1) \sum_{i=1}^{n} \nabla_{\beta}\left(\mathbf{z}_{i}\right) \\
& -(1+\theta) \sum_{i=1}^{n} \log \left\{\left[1-\nabla_{\beta}\left(\mathbf{z}_{i}\right)^{-\alpha}\right]^{v}+\nabla_{\beta}\left(\mathbf{z}_{i}\right)^{-\alpha v \theta}\right\} .
\end{aligned}
$$

The components of the score vector, $\mathbf{U}(\underline{\xi})=\frac{\partial \ell(\underline{\xi})}{\partial \underline{\xi}}=\left(\frac{\partial \ell(\underline{\xi})}{\partial v}, \frac{\partial \ell(\underline{\xi})}{\partial \theta}, \frac{\partial \ell(\underline{\xi})}{\partial \alpha}, \frac{\partial \ell(\underline{\xi})}{\partial \beta}\right)^{\top}$, are available if needed. Setting $\mathbf{U}(v)=\mathbf{U}(\theta)=\mathbf{U}(\alpha)=\mathbf{U}(\beta)=\mathbf{0}$ and solving them simultaneously yields the MLE $\underline{\xi}=(\widehat{v}, \widehat{\theta}, \widehat{\alpha}, \widehat{\beta}) \boldsymbol{\top}$. To solve these equations, it is usually more convenient to use nonlinear optimization methods such as the quasi-Newton algorithm to numerically maximize $\ell$. For interval estimation of the parameters, we obtain the $4 \times 4$ observed information matrix $\mathbf{J}(\underline{\xi})=\left\{\partial^{2} \ell(\underline{\xi}) / \partial m \partial w\right\} \operatorname{symbol}_{(m, w=v, \theta, \alpha, \beta)}$. 
4.1.2. OLS Let $F_{\underline{\xi}}\left(\mathrm{z}_{[i: n]}\right)$ denotes the CDF of OBP model and let $\mathrm{z}_{1}<\mathrm{z}_{2}<\cdots<\mathrm{z}_{n}$ be the $n$ ordered RS. The OLSEs are obtained upon minimizing

$$
\operatorname{OLSE}(\underline{\xi})=\sum_{i=1}^{n}\left[F_{\underline{\xi}}\left(\mathbf{z}_{[i: n]}\right)-\tau_{(i, n)}\right]^{2},
$$

then, we have

$$
\operatorname{OLSE}(\underline{\xi})=\sum_{i=1}^{n}\left(1-\frac{\nabla_{\beta}\left(\mathbf{z}_{[i: n]}\right)^{-\alpha v \theta}}{\left\{\left[1-\nabla_{\beta}\left(\mathbf{z}_{[i: n]}\right)^{-\alpha}\right]^{v}+\nabla_{\beta}\left(\mathbf{z}_{[i: n]}\right)^{-\alpha v}\right\}^{\theta}}-\tau_{(i, n)}\right)^{2}
$$

where $\tau_{(i, n)}=\frac{i}{n+1}$.The LSEs are obtained via solving the following non-linear equations

$$
\begin{aligned}
& 0=\sum_{i=1}^{n}\left(1-\frac{\nabla_{\beta}\left(\mathbf{z}_{[i: n]}\right)^{-\alpha v \theta}}{\left\{\left[1-\nabla_{\beta}\left(\mathbf{z}_{[i: n]}\right)^{-\alpha}\right]^{v}+\nabla_{\beta}\left(\mathbf{z}_{[i: n]}\right)^{-\alpha v}\right\}^{\theta}}-\tau_{(i, n)}\right) \varsigma_{v}\left(\mathbf{z}_{[i: n]}, \underline{\xi}\right), \\
& 0=\sum_{i=1}^{n}\left(1-\frac{\nabla_{\beta}\left(\mathbf{z}_{[i: n]}\right)^{-\alpha v \theta}}{\left\{\left[1-\nabla_{\beta}\left(\mathbf{z}_{[i: n]}\right)^{-\alpha}\right]^{v}+\nabla_{\beta}\left(\mathbf{z}_{[i: n]}\right)^{-\alpha v}\right\}^{\theta}}-\tau_{(i, n)}\right) \varsigma_{\theta}\left(\mathbf{z}_{[i: n]}, \underline{\xi}\right), \\
& 0=\sum_{i=1}^{n}\left(1-\frac{\nabla_{\beta}\left(\mathbf{z}_{[i: n]}\right)^{-\alpha v \theta}}{\left\{\left[1-\nabla_{\beta}\left(\mathbf{z}_{[i: n]}\right)^{-\alpha}\right]^{v}+\nabla_{\beta}\left(\mathbf{z}_{[i: n]}\right)^{-\alpha v}\right\}^{\theta}}-\tau_{(i, n)}\right) \varsigma_{\alpha}\left(\mathbf{z}_{[i: n]}, \underline{\xi}\right), \\
& 0=\sum_{i=1}^{n}\left(1-\frac{\nabla_{\beta}\left(\mathbf{z}_{[i: n]}\right)^{-\alpha v \theta}}{\left\{\left[1-\nabla_{\beta}\left(\mathbf{z}_{[i: n]}\right)^{-\alpha}\right]^{v}+\nabla_{\beta}\left(\mathbf{z}_{[i: n]}\right)^{-\alpha v}\right\}^{\theta}}-\tau_{(i, n)}\right) \varsigma_{\beta}\left(\mathbf{z}_{[i: n]}, \underline{\xi}\right),
\end{aligned}
$$

where $\varsigma_{v}\left(\mathbf{z}_{[i: n]}, \underline{\xi}\right), \varsigma_{\theta}\left(\mathbf{z}_{[i: n]}, \underline{\xi}\right), \varsigma_{\alpha}\left(\mathbf{z}_{[i: n]}, \underline{\xi}\right)$ and $\varsigma_{\beta}\left(\mathbf{z}_{[i: n]}, \underline{\xi}\right)$ defined above.

4.1.3. WLSE The WLSE are obtained by minimizing the function WLSE $(\underline{\xi})$ WRT $v, \theta, \alpha$ and $\beta$

$$
\operatorname{WLSE}(\underline{\xi})=\sum_{i=1}^{n} \omega_{(i, n)}\left[F_{\underline{\xi}}\left(\mathbf{z}_{[i: n]}\right)-\tau_{(i, n)}\right]^{2},
$$

where $\omega_{(i, n)}=\left[(1+n)^{2}(2+n)\right] /[i(1+n-i)]$. The WLSEs are obtained by solving

$$
\begin{aligned}
& 0=\sum_{i=1}^{n} \omega_{(i, n)}\left(1-\frac{\nabla_{\beta}\left(\mathbf{z}_{[i: n]}\right)^{-\alpha v \theta}}{\left\{\left[1-\nabla_{\beta}\left(\mathbf{z}_{[i: n]}\right)^{-\alpha}\right]^{v}+\nabla_{\beta}\left(\mathbf{z}_{[i: n]}\right)^{-\alpha v}\right\}^{\theta}}-\tau_{(i, n)}\right) \varsigma_{v}\left(\mathbf{z}_{[i: n]}, \underline{\xi}\right), \\
& 0=\sum_{i=1}^{n} \omega_{(i, n)}\left(1-\frac{\nabla_{\beta}\left(\mathbf{z}_{[i: n]}\right)^{-\alpha v \theta}}{\left\{\left[1-\nabla_{\beta}\left(\mathbf{z}_{[i: n]}\right)^{-\alpha}\right]^{v}+\nabla_{\beta}\left(\mathbf{z}_{[i: n]}\right)^{-\alpha v}\right\}^{\theta}}-\tau_{(i, n)}\right) \varsigma_{\theta}\left(\mathbf{z}_{[i: n]}, \underline{\xi}\right),
\end{aligned}
$$




$$
0=\sum_{i=1}^{n} \omega_{(i, n)}\left(1-\frac{\nabla_{\beta}\left(\mathbf{z}_{[i: n]}\right)^{-\alpha v \theta}}{\left\{\left[1-\nabla_{\beta}\left(\mathbf{z}_{[i: n]}\right)^{-\alpha}\right]^{v}+\nabla_{\beta}\left(\mathbf{z}_{[i: n]}\right)^{-\alpha v}\right\}^{\theta}}-\tau_{(i, n)}\right) \varsigma_{\alpha}\left(\mathbf{z}_{[i: n]}, \underline{\xi}\right),
$$

and

$$
0=\sum_{i=1}^{n} \omega_{(i, n)}\left(1-\frac{\nabla_{\beta}\left(\mathbf{z}_{[i: n]}\right)^{-\alpha v \theta}}{\left\{\left[1-\nabla_{\beta}\left(\mathbf{z}_{[i: n]}\right)^{-\alpha}\right]^{v}+\nabla_{\beta}\left(\mathbf{z}_{[i: n]}\right)^{-\alpha v}\right\}^{\theta}}-\tau_{(i, n)}\right) \varsigma_{\beta}\left(\mathbf{z}_{[i: n]}, \underline{\xi}\right)
$$

where $\varsigma_{v}\left(\mathbf{z}_{[i: n]}, \underline{\xi}\right), \varsigma_{\theta}\left(\mathbf{z}_{[i: n]}, \underline{\xi}\right), \varsigma_{\alpha}\left(\mathbf{z}_{[i: n]}, \underline{\xi}\right)$ and $\varsigma_{\beta}\left(\mathbf{z}_{[i: n]}, \underline{\xi}\right)$ defined above.

4.1.4. KE method The Kolmogorov estimates (KEs) $\widehat{v}, \widehat{\theta}, \widehat{\alpha}$ and $\widehat{\beta}$ and $\widehat{\beta}$ of $v, \theta, \alpha$ and $\beta$ are obtained by minimizing the function

$$
K E=K E(v, \theta, \alpha, \beta)=\max _{1 \leq i \leq n}\left\{\frac{i}{n}-F_{\underline{\xi}}\left(\mathbf{z}_{[i: n]}\right), F_{\underline{\xi}}\left(\mathbf{z}_{[i: n]}\right)-\frac{i-1}{n}\right\}
$$

\subsection{Bayesian estimation}

Assume the gamma priors of the parameters $v, \theta, \alpha$ and $\beta$ of the following forms

$$
\begin{gathered}
\pi_{1 ;\left(\zeta_{1}, \xi_{1}\right)}(v) \sim \operatorname{Gamma}\left(\zeta_{1}, \xi_{1}\right), \pi_{2 ;\left(\zeta_{2}, \xi_{2}\right)}(\theta) \sim \operatorname{Gamma}\left(\zeta_{2}, \xi_{2}\right) \\
\pi_{3 ;\left(\zeta_{3}, \xi_{3}\right)}(\alpha) \sim \operatorname{Gamma}\left(\zeta_{3}, \xi_{3}\right) \text { and } \pi_{4 ;\left(\zeta_{4}, \xi_{4}\right)}(\beta) \sim \operatorname{Uniform}\left(\zeta_{4}, \xi_{4}\right),
\end{gathered}
$$

Assume that the parameters are independently distributed. The joint prior distribution can be written as

$$
\begin{aligned}
\pi_{\left(\zeta_{i}, \xi_{i}\right)}(v, \theta, \alpha, \beta)= & \frac{\xi_{1}^{\zeta_{1}} \xi_{2}^{\zeta_{2}} \xi_{3}^{\zeta_{3}} \xi_{4}^{\zeta_{4}}}{\Gamma\left(\zeta_{1}\right) \Gamma\left(\zeta_{2}\right) \Gamma\left(\zeta_{3}\right) \Gamma\left(\zeta_{4}\right)} v^{\zeta_{1}-1} \theta^{\zeta_{2}-1} \alpha^{\zeta_{3}-1} \beta^{\zeta_{4}-1} \\
& \times \exp \left[-\left(v \xi_{1}+\theta \xi_{2}+\alpha \xi_{3}+\beta \xi_{4}\right)\right]
\end{aligned}
$$

The posterior distribution $\pi(v, \theta, \alpha, \beta \mid \underline{Z})$ of the parameters is defined as

$$
\pi(v, \theta, \alpha, \beta \mid \underline{Z}) \propto \operatorname{likelihood}(\underline{\xi} \mid \underline{Z}) \times \pi_{\left(\zeta_{i}, \xi_{i}\right)}(v, \theta, \alpha, \beta) .
$$

Under squared error loss function, the Bayesian estimators of $v, \theta, \alpha$ and $\beta$ are the means of their marginal posteriors and defined as

$$
\begin{gathered}
\widehat{v}_{\text {Bayesian }}=\int_{v, \theta, \alpha, \beta} v \pi(v, \theta, \alpha, \beta \mid \underline{Z}) d \beta d \alpha d \theta d v, \widehat{\theta}_{\text {Bayesian }}=\int_{\theta, \alpha, v, \beta} \theta \pi(v, \theta, \alpha, \beta \mid \underline{Z}) d \beta d v d \alpha d \theta, \\
\widehat{\alpha}_{\text {Bayesian }}=\int_{\alpha, \theta, v, \beta} \alpha \pi(v, \theta, \alpha, \beta \mid \underline{Z}) d \beta d v d \theta d \alpha \text { and } \widehat{\beta}_{\text {Bayesian }}=\int_{\beta, v, \theta, \alpha} \beta \pi(v, \theta, \alpha, \beta \mid \underline{Z}) d \alpha d \theta d v d \beta,
\end{gathered}
$$

respectively. It is not possible to obtain the Bayesian estimates through the above formulae. So, the numerical approximation are needed. We propose the use of MCMC techniques namely Gibbs sampler and Metropolis Hastings (M-H) algorithm. Since the conditional posteriors of the parameters $v, \theta, \alpha$ and $\beta$ cannot be obtained in any standard forms, therefore, using a hybrid MCMC for drawing samples from the joint posterior of the parameters is suggested. the full conditional posteriors of $v, \theta, \alpha$ and $\beta$ can be easily derived. The simulation algorithm is given by

1. Provide the initial values, say $v, \theta, \alpha$ and $\beta$ then at $i^{(\text {th })}$ stage,

2. Using M-H algorithm, we generate $v_{(i)} \sim \pi_{1}\left(\left.v_{(i)}\right|_{\theta_{(i)}, \alpha_{(i)}, \beta_{(i)}}, \underline{Z}\right)$;

3. Using M-H algorithm, we generate $\theta_{(i)} \sim \pi_{2}\left(\left.\theta\right|_{v_{(i)}, \alpha_{(i)}, \beta_{(i)}}, \underline{Z}\right)$; 
4. Using M-H algorithm, we generate $\alpha_{(i)} \sim \pi_{2}\left(\left.\alpha\right|_{v_{(i)}, \theta_{(i)}, \beta_{(i)}}, \underline{Z}\right)$;

5. Using M-H algorithm, we generate $\beta_{(i)} \sim \pi_{3}\left(\left.\beta\right|_{v_{(i)}, \theta_{(i)}, \alpha_{(i)}}, \underline{Z}\right)$;

6. Repeat steps $2-5, \mathbf{M}=\mathbf{1 0 0 0 0 0}$ times to get the samples of size $M$ from the corresponding posteriors of interest. Obtain the Bayesian estimates of $v, \theta, \alpha$ and $\beta$ using the following formulae

$$
\begin{gathered}
\widehat{v}_{(\text {Bayesian })}=\frac{1}{\mathbf{M}-\mathbf{M}_{0}} \sum_{h=M_{0}+1}^{M} v^{[h]}, \widehat{\theta}_{(\text {Bayesian })}=\frac{1}{\mathbf{M}-\mathbf{M}_{0}} \sum_{h=M_{0}+1}^{M} \theta^{[h]} \\
\widehat{\alpha}_{(\text {Bayesian })}=\frac{1}{\mathbf{M}-\mathbf{M}_{0}} \sum_{h=M_{0}+1}^{M} \alpha^{[h]} \text { and } \widehat{\beta}_{(\text {Bayesian })}=\frac{1}{\mathbf{M}-\mathbf{M}_{0}} \sum_{h=M_{0}+1}^{M} \beta^{[h]},
\end{gathered}
$$

respectively, where $\mathbf{M}_{0}(\approx \mathbf{5 0 0 0 0})$ is the burn-in period of the generated MCMC.

\section{Simulation studies for comparing estimation methods}

A numerical simulation is performed in to compare the classical estimation methods. The simulation study is based on $\mathrm{N}=1000$ generated data sets from the OBP version where $n=50,100,150$ and 300 and

$\begin{array}{lllll} & \alpha & \beta & \theta & v \\ \text { I } & 0.6 & 0.5 & 0.8 & 2.0 \\ \text { II } & 1.2 & 0.9 & 0.4 & 0.9 \\ \text { III } & 1.5 & 1.5 & 1.5 & 1.5\end{array}$

The estimates are compared in terms of their

1-Bias $\operatorname{BIAS}(\xi)$;

2-Root mean-standard error $\operatorname{RMSE}(\xi)$;

3 -the mean of the absolute difference between the theoretical and the estimates "D-abs" and

4-the maximum absolute difference between the true parameters and estimates "D-max". Where

$$
\begin{aligned}
\operatorname{BIAS}(v) & =\frac{1}{B} \sum_{i=1}^{\theta}\left(\widehat{v}_{i}-v\right), \quad \operatorname{BIAS}(\theta)=\frac{1}{B} \sum_{i=1}^{B}\left(\widehat{\theta}_{i}-\theta\right), \\
\operatorname{BIAS}(\alpha) & =\frac{1}{B} \sum_{i=1}^{B}\left(\widehat{\alpha}_{i}-\alpha\right) \text { and } \operatorname{BIAS}(\beta)=\frac{1}{B} \sum_{i=1}^{B}\left(\widehat{\beta}_{i}-\beta\right) \\
\operatorname{RMSE}(v) & =\sqrt{\frac{1}{B} \sum_{i=1}^{B}\left(\widehat{v}_{i}-v\right)^{2},} \operatorname{RMSE}(\theta)=\sqrt{\frac{1}{B} \sum_{i=1}^{B}\left(\widehat{\theta}_{i}-\theta\right)^{2}}, \\
\operatorname{RMSE}(\alpha) & =\sqrt{\frac{1}{B} \sum_{i=1}^{B}\left(\widehat{\alpha}_{i}-\alpha\right)^{2}} \text { and } \operatorname{RMSE}(\beta)=\sqrt{\frac{1}{B} \sum_{i=1}^{B}\left(\widehat{\beta}_{i}-\beta\right)^{2}} \\
\text { D-abs } & =\frac{1}{n B} \sum_{i=1}^{B} \sum_{j=1}^{n}\left|F_{\widehat{v}, \widehat{\theta}, \widehat{\alpha}, \widehat{\beta}}\left(w_{i j}\right)-F_{\widehat{v}, \widehat{\theta}, \widehat{\alpha}, \widehat{\beta}}\left(t_{i j}\right)\right|
\end{aligned}
$$

and

$$
\mathrm{D}-\max =\frac{1}{B} \sum_{i=1}^{B} \max _{j}\left|F_{\widehat{v}, \widehat{\theta}, \widehat{\alpha}, \widehat{\beta}}\left(w_{i j}\right)-F_{\widehat{v}, \widehat{\theta}, \widehat{\alpha}, \widehat{\beta}}\left(t_{i j}\right)\right| .
$$


From Tables 2, 3 and 4 we note that:

1-The BIAS $(\xi)$ tend to zero when $\mathrm{n}$ increases which means that all estimators are non-biased.

2-The $\operatorname{RMSE}(\underline{\xi})$ tend to zero when $\mathrm{n}$ increases which means incidence of consistency property.

Table 2: Simulation results for blend I.

\begin{tabular}{|c|c|c|c|c|c|c|c|c|c|c|c|}
\hline & \multirow[t]{2}{*}{$\mathrm{n}$} & \multicolumn{4}{|c|}{ BIAS } & \multicolumn{4}{|c|}{ RMSE } & \multicolumn{2}{|c|}{$\mathrm{D}$} \\
\hline & & $\alpha$ & $\beta$ & $\theta$ & $\nu$ & $\alpha$ & $\beta$ & $\theta$ & $\nu$ & D-abs & D-max \\
\hline$\overline{\overline{\text { MLE }}}$ & $\overline{50}$ & 0.00080 & 0.01097 & 0.00975 & 0.02018 & 0.05360 & $\overline{0.08511}$ & 0.11800 & 0.25189 & 0.00287 & 0.00512 \\
\hline OLS & & 0.00023 & 0.01038 & 0.00560 & -0.08725 & 0.05526 & 0.08647 & 0.13487 & 0.30543 & 0.00728 & 0.01358 \\
\hline WLS & & 0.00500 & 0.02796 & 0.00893 & 0.01640 & 0.11195 & 0.15991 & 0.30269 & 0.10696 & 0.00863 & 0.01475 \\
\hline $\mathrm{KE}$ & & 0.01374 & -0.00721 & 0.04123 & -0.01073 & 0.05907 & 0.08539 & 0.14970 & 0.38113 & 0.02621 & 0.03798 \\
\hline Bayes & & 0.00398 & 0.01568 & 0.03254 & 0.33775 & 0.05745 & 0.08026 & 0.10377 & 0.36938 & 0.02256 & 0.03717 \\
\hline MLE & 100 & 0.00129 & 0.00535 & 0.00798 & 0.02097 & 0.03744 & 0.05787 & 0.08208 & 0.17399 & 0.00248 & 0.00475 \\
\hline OLS & & 0.00065 & 0.00460 & 0.00441 & -0.03183 & 0.03869 & 0.05907 & 0.09347 & 0.21176 & 0.00211 & 0.00379 \\
\hline WLS & & 0.00712 & 0.01080 & 0.01685 & 0.01777 & 0.07897 & 0.11146 & 0.21620 & 0.07382 & 0.00448 & 0.00781 \\
\hline $\mathrm{KE}$ & & 0.00707 & -0.00421 & 0.02055 & 0.02126 & 0.04024 & 0.06001 & 0.09900 & 0.28228 & 0.01430 & 0.02181 \\
\hline Bayes & & 0.00729 & 0.04256 & 0.07521 & 0.14118 & 0.03976 & 0.07488 & 0.09686 & 0.19151 & 0.01959 & 0.03429 \\
\hline MLE & 150 & 0.00156 & 0.00211 & 0.00596 & 0.01141 & 0.02993 & 0.04646 & 0.06472 & 0.13968 & 0.00235 & 0.00441 \\
\hline OLS & & 0.00141 & 0.00146 & 0.00508 & -0.02001 & 0.03124 & 0.04715 & 0.07501 & 0.016616 & 0.00154 & 0.00307 \\
\hline WLS & & 0.00536 & 0.00568 & 0.01342 & 0.01155 & 0.06129 & 0.08744 & 0.16899 & 0.05827 & 0.00273 & 0.00426 \\
\hline $\mathrm{KE}$ & & 0.00582 & -0.00486 & 0.01658 & 0.00590 & 0.03221 & 0.04760 & 0.07936 & 0.21698 & 0.01210 & 0.01784 \\
\hline Bayes & & -0.00358 & -0.00315 & -0.05250 & -0.05662 & 0.03878 & 0.04345 & 0.08228 & 0.19074 & 0.01916 & 0.03325 \\
\hline MLE & 300 & 0.00017 & 0.00194 & 0.00185 & 0.00588 & 0.02114 & 0.03265 & 0.04585 & 0.10084 & 0.00065 & 0.00106 \\
\hline OLS & & -0.00021 & 0.00198 & 0.00027 & -0.01669 & 0.02224 & 0.03364 & 0.05306 & 0.12376 & 0.00170 & 0.00319 \\
\hline WLS & & 0.00088 & 0.00659 & 0.00156 & 0.00813 & 0.04187 & 0.06288 & 0.11659 & 0.04153 & 0.00321 & 0.00563 \\
\hline $\mathrm{KE}$ & & 0.00219 & -0.00113 & 0.00646 & -0.00210 & 0.02282 & 0.03408 & 0.05580 & 0.15528 & 0.00417 & 0.00603 \\
\hline Bayes & & 0.03087 & -0.03077 & 0.04631 & 0.16941 & 0.03068 & 0.04330 & 0.06821 & 0.12107 & 0.00580 & 0.00917 \\
\hline
\end{tabular}

Table 3: Simulation results for blend II.

\begin{tabular}{|c|c|c|c|c|c|c|c|c|c|c|c|}
\hline & \multirow[t]{2}{*}{$\mathrm{n}$} & \multicolumn{4}{|c|}{ BIAS } & \multicolumn{4}{|c|}{ RMSE } & \multicolumn{2}{|c|}{$\mathrm{D}$} \\
\hline & & $\alpha$ & $\beta$ & $\theta$ & $\nu$ & $\alpha$ & $\beta$ & $\theta$ & $\nu$ & D-abs & D-max \\
\hline MLE & 50 & 0.02788 & 0.05455 & 0.00880 & 0.01173 & 0.18563 & 0.35095 & 0.06044 & 0.11516 & 0.00683 & 0.01350 \\
\hline OLS & & 0.02315 & 0.05498 & 0.00709 & 0.00706 & 0.21622 & 0.36186 & 0.06881 & 0.16892 & 0.00442 & 0.00913 \\
\hline WLS & & 0.01899 & 0.05403 & 0.00596 & 0.02253 & 0.19359 & 0.36204 & 0.06252 & 0.13636 & 0.00653 & 0.01262 \\
\hline $\mathrm{KE}$ & & 0.07836 & -0.01383 & 0.02427 & 0.02780 & 0.24575 & 0.33801 & 0.07720 & 0.18985 & 0.03616 & 0.05639 \\
\hline Bayes & & -0.19824 & 0.00974 & 0.01411 & 0.04312 & 0.24994 & 0.21532 & 0.06691 & 0.11820 & 0.05550 & 0.03858 \\
\hline MLE & 100 & 0.01000 & 0.03649 & 0.00307 & 0.00650 & 0.12325 & 0.24593 & 0.04019 & 0.08044 & 0.00226 & 0.00404 \\
\hline OLS & & 0.00176 & 0.04617 & 0.00038 & -0.00230 & 0.14635 & 0.25991 & 0.04682 & 0.11518 & 0.00534 & 0.00828 \\
\hline WLS & & 0.00513 & 0.05259 & 0.00143 & 0.01957 & 0.13272 & 0.26865 & 0.04309 & 0.09157 & 0.00387 & 0.00691 \\
\hline $\mathrm{KE}$ & & 0.02951 & 0.01036 & 0.00910 & 0.01022 & 0.16007 & 0.25481 & 0.05060 & 0.13287 & 0.01190 & 0.01917 \\
\hline Bayes & & -0.05279 & -0.16918 & 0.05055 & 0.08384 & 0.19302 & 0.21199 & 0.05662 & 0.10322 & 0.03506 & 0.08749 \\
\hline MLE & 150 & 0.00263 & 0.02757 & 0.00074 & 0.00202 & 0.09923 & 0.19196 & 0.03234 & 0.06614 & 0.00206 & 0.00372 \\
\hline OLS & & 0.00679 & 0.02059 & 0.00206 & 0.00268 & 0.11844 & 0.19843 & 0.03791 & 0.09320 & 0.00128 & 0.00249 \\
\hline WLS & & 0.00848 & 0.02724 & 0.00260 & 0.01843 & 0.10600 & 0.21046 & 0.03447 & 0.07537 & 0.00441 & 0.00830 \\
\hline $\mathrm{KE}$ & & 0.02476 & -0.00370 & 0.00769 & 0.01246 & 0.12804 & 0.20073 & 0.04054 & 0.10438 & 0.01223 & 0.01942 \\
\hline Bayes & & -0.17180 & -0.01111 & 0.00525 & -0.01755 & 0.16913 & 0.20290 & 0.05171 & 0.06349 & 0.03470 & 0.05529 \\
\hline MLE & 300 & 0.00510 & 0.01484 & 0.00153 & 0.00399 & 0.07060 & 0.13355 & 0.02300 & 0.04620 & 0.00133 & 0.00260 \\
\hline OLS & & 0.00103 & 0.01383 & 0.00027 & -0.00042 & 0.08214 & 0.13814 & 0.02635 & 0.06489 & 0.00138 & 0.00222 \\
\hline WLS & & 0.00370 & 0.01881 & 0.00109 & 0.01164 & 0.07459 & 0.14737 & 0.02428 & 0.05348 & 0.00233 & 0.00417 \\
\hline KE & & 0.00918 & 0.00284 & 0.00284 & 0.00324 & 0.08606 & 0.13878 & 0.02734 & 0.06999 & 0.00379 & 0.00610 \\
\hline Bayes & & -0.09043 & -0.19675 & 0.04463 & -0.00204 & 0.11547 & 0.09998 & 0.03676 & 0.04062 & 0.02906 & 0.05239 \\
\hline
\end{tabular}


Table 4: Simulation results for blend III.

\begin{tabular}{|c|c|c|c|c|c|c|c|c|c|c|c|}
\hline & \multirow[t]{2}{*}{$\mathrm{n}$} & \multicolumn{4}{|c|}{ BIAS } & \multicolumn{4}{|c|}{ RMSE } & \multicolumn{2}{|c|}{$\mathrm{D}$} \\
\hline & & $\alpha$ & $\beta$ & $\theta$ & $\nu$ & $\alpha$ & $\beta$ & $\theta$ & $\nu$ & D-abs & D-max \\
\hline MLE & 50 & 0.00998 & 0.01608 & 0.02478 & 0.02660 & 0.15515 & 0.20023 & 0.22342 & 0.17201 & 0.00356 & 0.00692 \\
\hline OLS & & 0.00977 & 0.01309 & 0.02195 & -0.02893 & 0.16803 & 0.20343 & 0.25625 & 0.24265 & 0.00524 & 0.00886 \\
\hline WLS & & 0.00878 & 0.01192 & 0.01731 & 0.03202 & 0.15788 & 0.20076 & 0.22766 & 0.20061 & 0.00374 & 0.00647 \\
\hline $\mathrm{KE}$ & & 0.05577 & -0.03849 & 0.09372 & -0.02485 & 0.18571 & 0.20171 & 0.29133 & 0.28028 & 0.03834 & 0.05543 \\
\hline Bayes & & 0.29424 & -0.36041 & 0.14774 & -0.03986 & 0.30536 & 0.38261 & 0.22526 & 0.17783 & 0.17937 & 0.25659 \\
\hline MLE & 100 & 0.00390 & 0.01037 & 0.01150 & 0.01402 & 0.11173 & 0.14382 & 0.15891 & 0.12593 & 0.00170 & 0.00264 \\
\hline OLS & & 0.01089 & -0.00168 & 0.01969 & -0.01795 & 0.11359 & 0.13804 & 0.17148 & 0.16423 & 0.00746 & 0.01083 \\
\hline WLS & & 0.00983 & -0.00038 & 0.01639 & 0.03038 & 0.10863 & 0.13984 & 0.15400 & 0.13601 & 0.00457 & 0.00916 \\
\hline $\mathrm{KE}$ & & 0.02399 & -0.01490 & 0.04074 & -0.01276 & 0.12439 & 0.14499 & 0.19049 & 0.20399 & 0.01651 & 0.02378 \\
\hline Bayes & & 0.05525 & -0.15882 & 0.07136 & -0.12017 & 0.13964 & 0.17427 & 0.13996 & 0.13171 & 0.06436 & 0.09694 \\
\hline MLE & 150 & 0.00633 & 0.00049 & 0.01217 & 0.00479 & 0.08774 & 0.11118 & 0.12507 & 0.10424 & 0.00319 & 0.00519 \\
\hline OLS & & -0.00200 & 0.01173 & -0.00024 & 0.00260 & 0.09947 & 0.12169 & 0.14955 & 0.14502 & 0.00311 & 0.00456 \\
\hline WLS & & 0.00009 & 0.01013 & 0.00255 & 0.03159 & 0.09527 & 0.12312 & 0.13427 & 0.11760 & 0.00432 & 0.00810 \\
\hline KE & & 0.01201 & -0.00571 & 0.02084 & -0.00562 & 0.09777 & 0.11529 & 0.14826 & 0.16543 & 0.00796 & 0.01147 \\
\hline Bayes & & -0.03666 & 0.11917 & -0.06103 & -0.00945 & 0.08742 & 0.15873 & 0.12070 & 0.08974 & 0.04253 & 0.06291 \\
\hline MLE & 300 & 0.00212 & 0.00192 & 0.00446 & 0.00532 & 0.06218 & 0.08054 & 0.08712 & 0.07095 & 0.00081 & 0.00162 \\
\hline OLS & & 0.00253 & 0.00103 & 0.00495 & -0.00464 & 0.06707 & 0.08203 & 0.10040 & 0.10000 & 0.00153 & 0.00226 \\
\hline WLS & & 0.00436 & -0.00045 & 0.00722 & 0.02002 & 0.06465 & 0.08327 & 0.09059 & 0.08067 & 0.00245 & 0.00469 \\
\hline $\mathrm{KE}$ & & 0.00768 & -0.00487 & 0.01296 & -0.00103 & 0.06878 & 0.08250 & 0.10397 & 0.11651 & 0.00514 & 0.00751 \\
\hline Bayes & & 0.02506 & -0.04209 & 0.05482 & 0.02469 & 0.06617 & 0.08058 & 0.10813 & 0.08053 & 0.02287 & 0.03505 \\
\hline
\end{tabular}

\section{Applications for comparing classical methods}

\subsection{Comparing classical methods under failure times}

The first real data set (data set $\mathbf{I}$ ) represents the data on failure times of 84 aircraft windshield given in Murthy et al. [58]. The data are: $0.0400,1.866,2.3850,3.443,0.3010,1.876,2.4810,3.467,0.309,1.8990,2.610,3.4780$, $0.557,1.9110,2.625,3.5780,0.943,1.9120,2.632,3.5950,1.0700,1.914,2.6460,3.699,1.1240,1.981,2.661$, $3.7790,1.248,2.0100,2.688,3.9240,1.2810,2.038,2.820,3,4.035,1.281,2.0850,2.890,4.121,1.3030,2.089$, $2.902,4.167,1.4320,2.097,2.934,4.2400,1.480,2.135,2.962,4.2550,1.505,2.154,2.9640,4.278,1.506,2.190$, $3.000,4.3050,1.568,2.1940,3.103,4.376,1.615,2.2230,3.114,4.449,1.6190,2.224,3.1170,4.485,1.652$, 2.2290, 3.166, 4.570, 1.652, 2.3000, 3.344, 4.602, 1.7570, 2.324, 3.3760, 4.663. We consider the Cramér-Von Mises $\left(\mathrm{W}^{\star}\right)$ and the Anderson-Darling $\left(\mathrm{A}^{\star}\right)$ statistis. Figure 6 gives probability-probability (P-P) plots for comparing all methods under the failure times data set. From Table 5, the MLE method is the best method with $\mathrm{W}^{\star}=0.05840$ and $\mathrm{A}^{\star}=0.56749$ then Bayesian method with $\mathrm{W}^{\star}=0.06309$ and $\mathrm{A}^{\star}=0.63239$.

Table 5: The values of estimators $\mathrm{A}^{\star}$ and $\mathrm{W}^{\star}$ under failure times.

\begin{tabular}{lcccccc}
\hline \hline Method & $\widehat{v}$ & $\widehat{\theta}$ & $\widehat{\alpha}$ & $\widehat{\beta}$ & $\mathrm{A}^{\star}$ & $\mathrm{W}^{\star}$ \\
\hline \hline MLE & $\mathbf{2 . 2 3 3 8 6}$ & $\mathbf{3 2 . 8 4 1 6 0}$ & $\mathbf{1 7 . 5 4 3 8 0}$ & $\mathbf{2 6 2 . 2 0 8}$ & $\mathbf{0 . 5 6 7 4 9}$ & $\mathbf{0 . 0 5 8 4 0}$ \\
OLS & 3.30660 & 1.24718 & 0.94249 & 2.45219 & 1.24500 & 0.14386 \\
WLS & 3.77635 & 1.92859 & 0.90976 & 2.63173 & 0.82385 & 0.08386 \\
KE & 2.65987 & 1.56671 & 1.28125 & 3.97197 & 1.08694 & 0.12031 \\
Bayes & 2.03701 & 43.35538 & 5.03022 & 97.70641 & 0.63239 & 0.06309 \\
\hline \hline
\end{tabular}

\subsection{Comparing classical methods under service times}

The second real data set (data set II) represents the data on service times of 63 aircraft windshield given in Murthy et al. [58]. The data are: $0.046,1.436,2.592,0.140,1.492,2.600,0.150,1.580,2.670,0.248,1.7190,2.717,0.2800$, $1.794,2.819,0.3130,1.915,2.820,0.389,1.9200,2.878,0.487,1.9630,2.950,0.622,1.978,3.0030,0.9000,2.053$, $3.1020,0.952,2.065,3.3040,0.9960,2.117,3.483,1.0030,2.137,3.500,1.0100,2.141,3.6220,1.085,2.163$, 
$3.6650,1.092,2.183,3.695,1.1520,2.2400,4.015,1.183,2.3410,4.628,1.2440,2.435,4.806,1.249,2.4640$, 4.881, 1.262, 2.5430, 5.140. Many other useful real life data sets can be found in Aryal et al. [18] and Ibrahim and Yousof [36]. Figure 7 gives P-P plots for comparing all methods under the failure times data set. From Table 6, the MLE method is the best method with $\mathrm{W}^{\star}=0.09443$ and $\mathrm{A}^{\star}=0.57283$ then the OLS method with $\mathrm{W}^{\star}=0.09443$ and $\mathrm{A}^{\star}=0.71474$.

Table 6: The values of estimators $A^{\star}$ and $W^{\star}$ under service times data.

\begin{tabular}{llccccc}
\hline \hline Method & \multicolumn{1}{c}{$\widehat{v}$} & $\widehat{\theta}$ & \multicolumn{1}{c}{$\widehat{\alpha}$} & $\widehat{\beta}$ & $\mathrm{A}^{\star}$ & $\mathrm{W}^{\star}$ \\
\hline \hline MLE & $\mathbf{1 . 5 7 8 7 7}$ & $\mathbf{1 7 . 0 7 3 9}$ & $\mathbf{7 7 . 3 1 3 1 9}$ & $\mathbf{1 1 5 0 . 4 7}$ & $\mathbf{0 . 5 7 2 8 3}$ & $\mathbf{0 . 0 9 4 4 3}$ \\
OLS & 1.176663 & 5.15567 & 1.59013 & 9.75342 & 0.71474 & 0.11726 \\
WLS & 1.89100 & 5.97335 & 0.92384 & 5.14160 & 0.78311 & 0.12841 \\
ME & 2.16031 & 1.38096 & 1.07536 & 2.52004 & 1.45874 & 0.23917 \\
Bayes & 1.33126 & 304.370 & 0.93765 & 155.711 & 0.84289 & 0.13915 \\
\hline \hline
\end{tabular}

\section{Graphical assessment for the MLEs}

Graphically and using the biases and mean squared errors (MSEs), we can perform the simulation experiments to assess the finite sample behavior of the MLEs. The assessment was based on $n=1000$ replication for all $\left.n\right|_{(n=50,100, \ldots, 500)}$. The following algorithm is considered:

1. Generate $n=1000$ samples of size $\left.n\right|_{(n=50,100, \ldots, 500)}$ from the OBP distribution using (7);

2. Compute the MLEs for the $n=1000$ samples, say

$$
\left.\left[\widehat{v_{n}}, \widehat{\theta_{n}}, \widehat{\alpha_{n}}, \widehat{\beta_{n}}\right]\right|_{(n=1,2, \ldots, 1000)},
$$

3. Compute the SEs of the MLEs for the 1000 samples, say

$$
\left.\left[S_{\widehat{v_{n}}}, S_{\widehat{\theta_{n}}}, S_{\widehat{\alpha_{n}}}, S_{\widehat{\beta_{n}}}\right]\right|_{(n=1,2, \ldots, 1000)} \text {. }
$$

The standard errors (SEs) were computed by inverting the observed information matrix.

4. Compute the biases and mean squared errors given for $\underline{\xi}=v, \theta, \alpha, \beta$. We repeated these steps for $\left.n\right|_{(n=50,100, \ldots, 500)}$ with $v=\theta=\alpha=\beta=1$, so computing $\operatorname{biases}\left(\operatorname{Bias}_{\underline{\xi}}(n)\right)$, mean squared errors (MSEs) $\left(\operatorname{MSE}_{h}(n)\right)$ for $\underline{\xi}=v, \theta, \alpha, \beta$ and $\left.n\right|_{(n=50,100, \ldots, 500)}$ where

$$
\left.\operatorname{Bias}_{\underline{\xi}}(n)\right|_{(\underline{\xi}=v, \theta, \alpha, \beta)}=\frac{1}{1000} \sum_{n=1}^{1000}\left(\underline{\widehat{\xi}}_{n}-\underline{\xi}\right),
$$

and

$$
\left.\operatorname{MSE}_{\underline{\xi}}(n)\right|_{(\underline{\xi}=v, \theta, \alpha, \beta)}=\frac{1}{1000} \sum_{n=1}^{1000}\left(\underline{\widehat{\xi}}_{n}-\underline{\xi}\right)^{2} .
$$

Figures 8, 9, 10 and 11 gives the biases (left panels) and MSEs (right panels) for the parameters $v, \theta, \alpha$ and $\beta$ respectively.

The left panels from show how the four biases vary with respect to $n$. The right panels show how the four MSEs vary with respect to $n$. The broken line in red in Figure 8 corresponds to the biases being 0 . From Figures 8, 9, 10 and 11 ( left panels), the biases for each parameter are generally negative and tends to zero as $n \rightarrow \infty$. From Figures 8, 9, 10 and 11 (right panels), the MSEs for each parameter decrease to zero as $n$ $\rightarrow \infty$. 


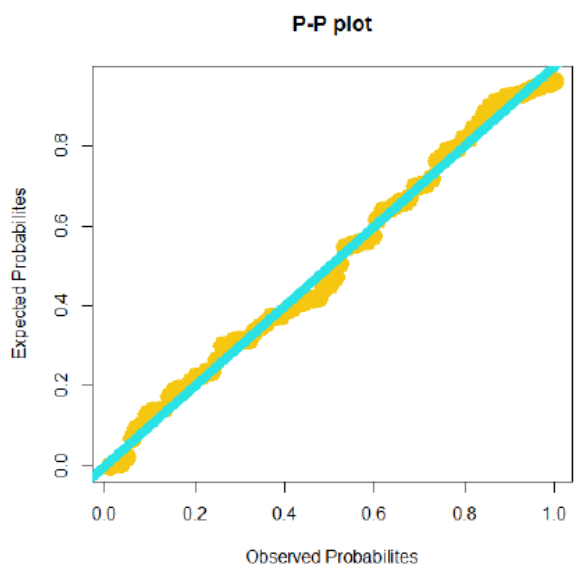

(a): MLE

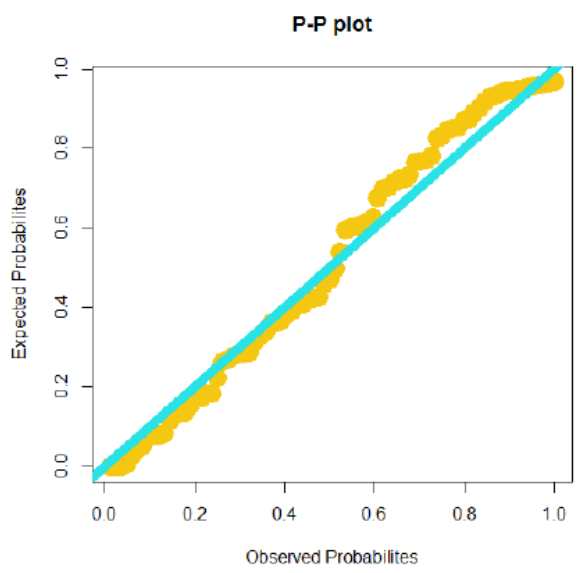

(c): WLS

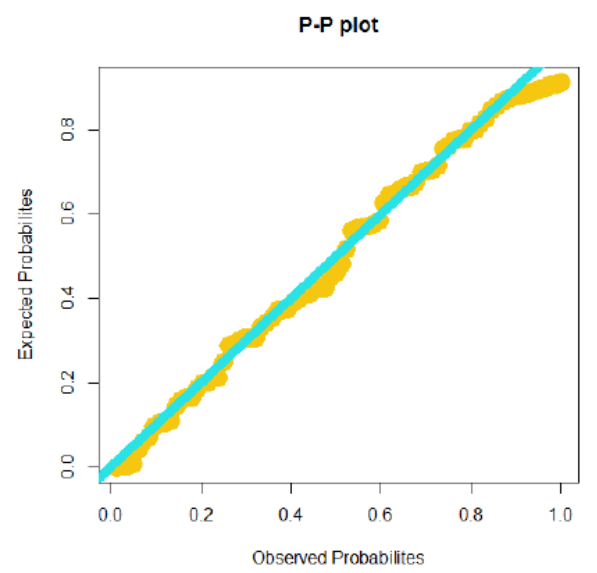

(b): OLS

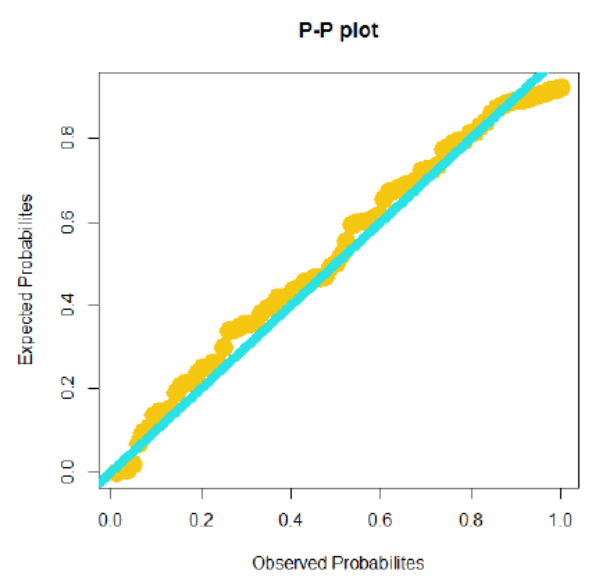

(d): KE

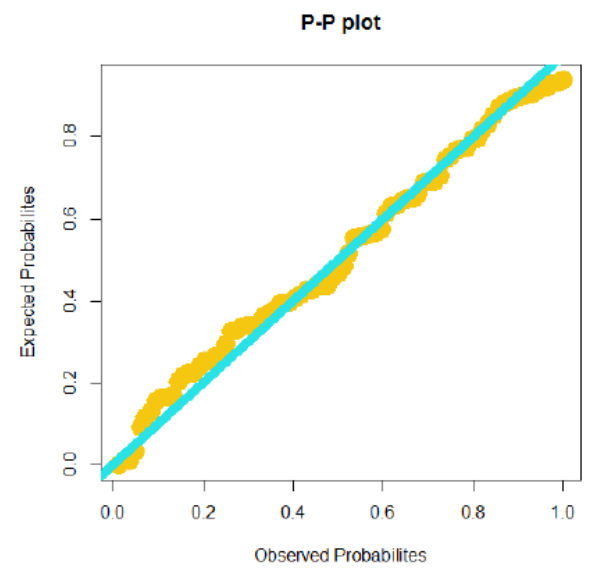

(e): Bayes

Figure 8. P-P plots for comparing classical methodsunder failure times. 


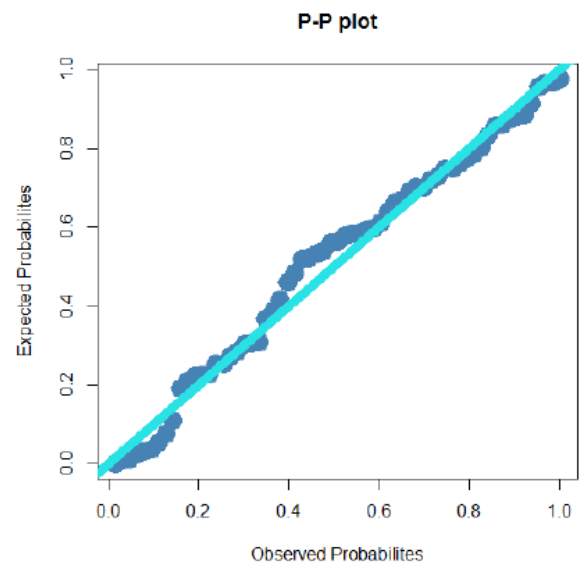

(a): MLE

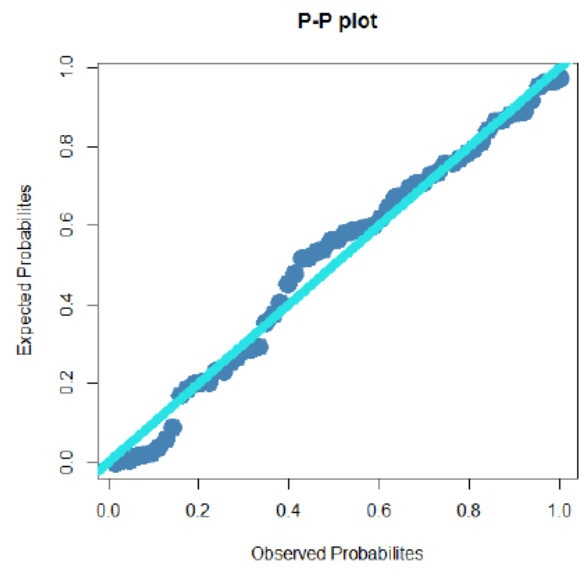

(c): WLS

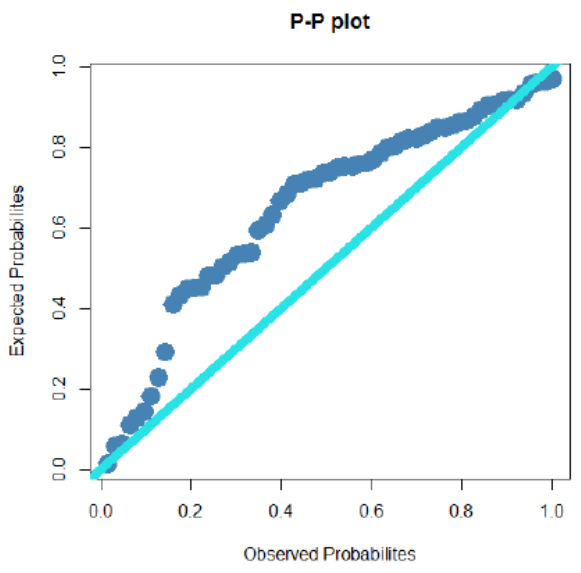

(b): OLS

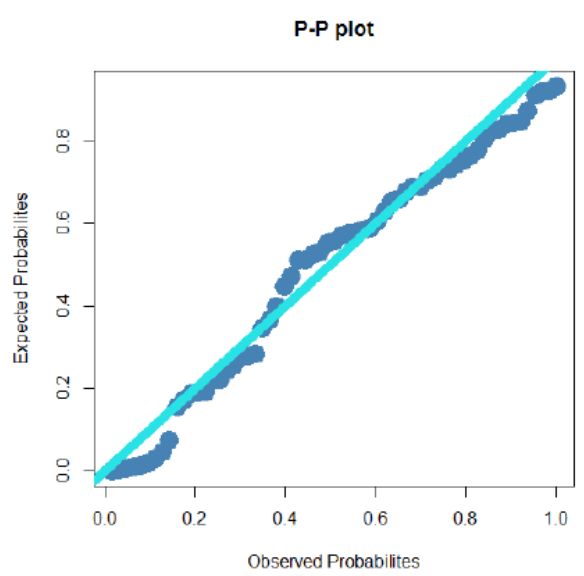

(d): KE

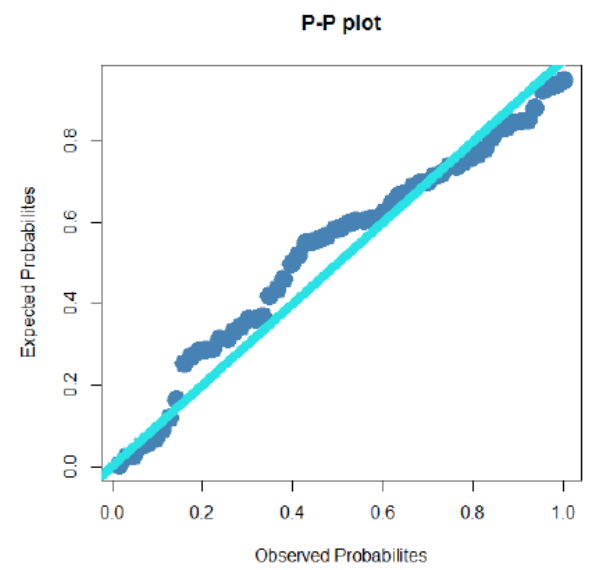

(e): Bayes

Figure 9. P-P plots for comparing classical methods under service times data 

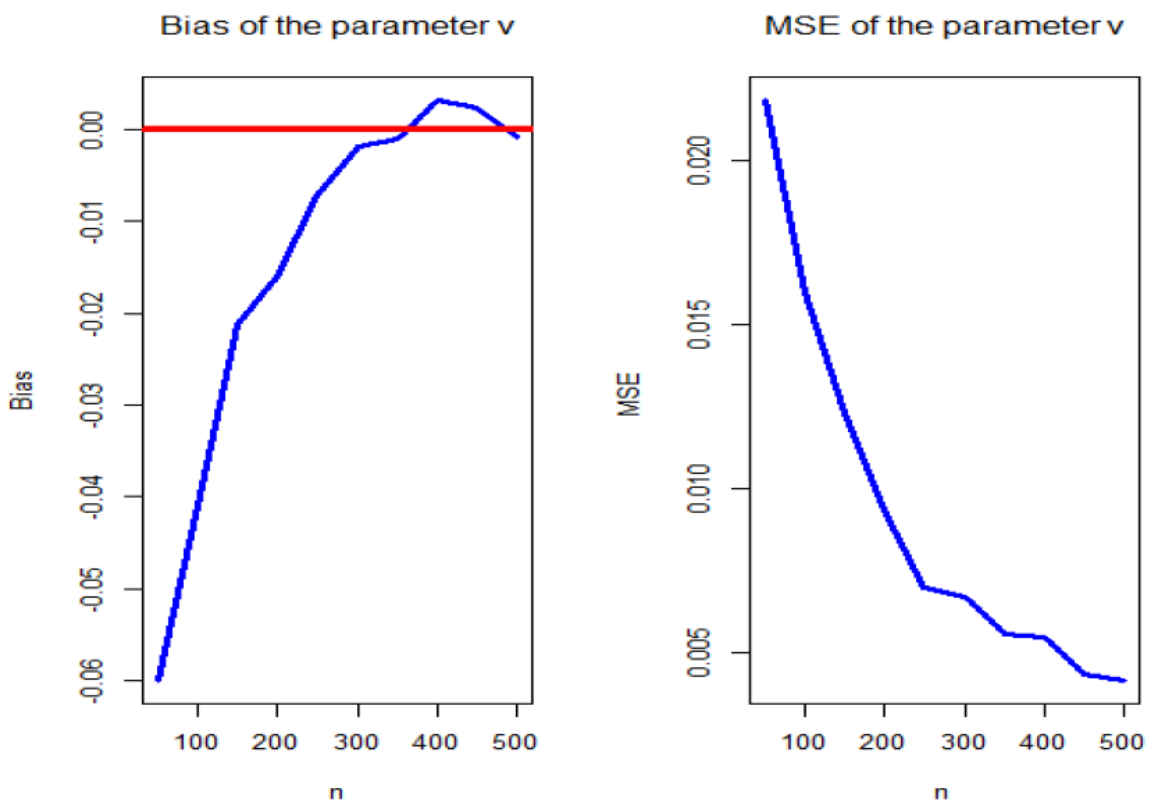

Figure 10. biases and mean squared errors for the parameter v.
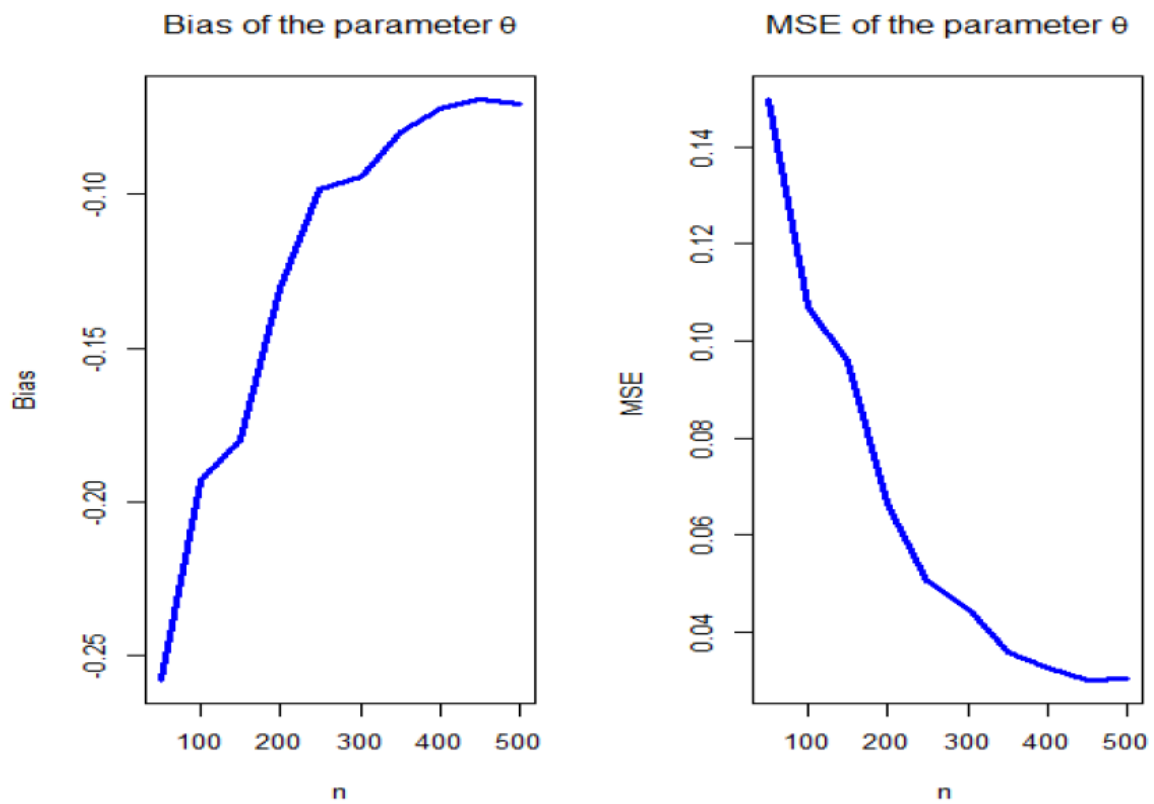

Figure 11. biases and mean squared errors for the parameter $\theta$. 
Bias of the parameter $\alpha$

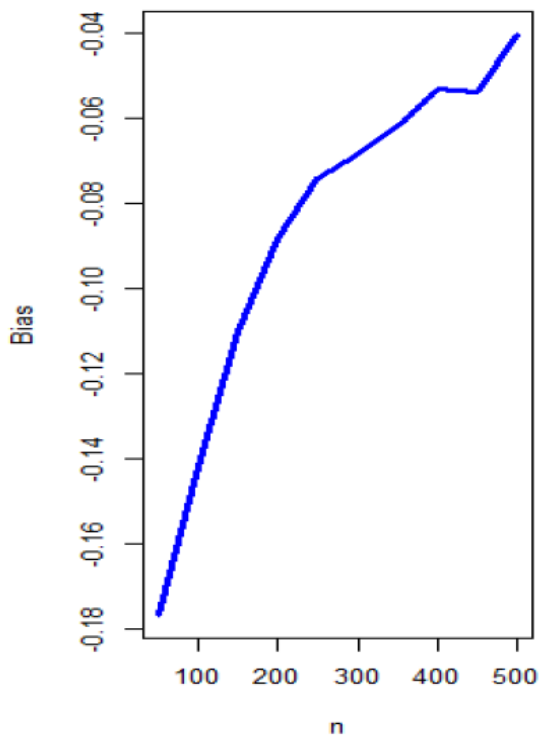

MSE of the parameter $\alpha$

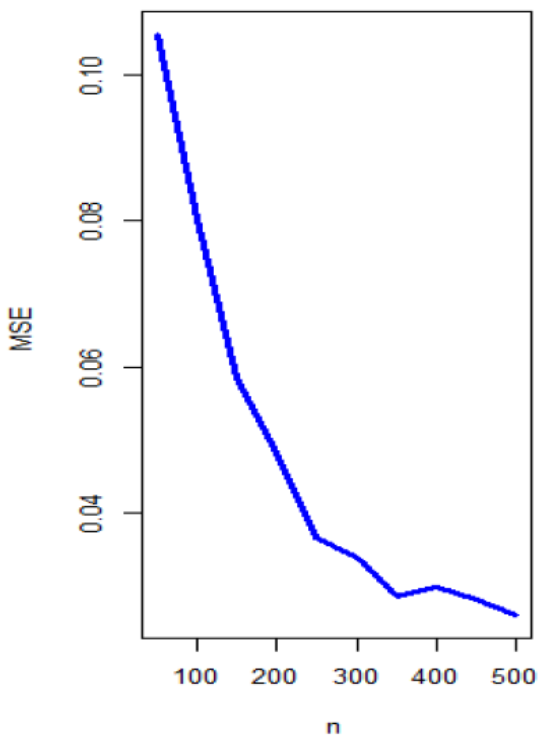

Figure 12. biases and mean squared errors for the parameter $\alpha$.

Bias of the parameter $\beta$

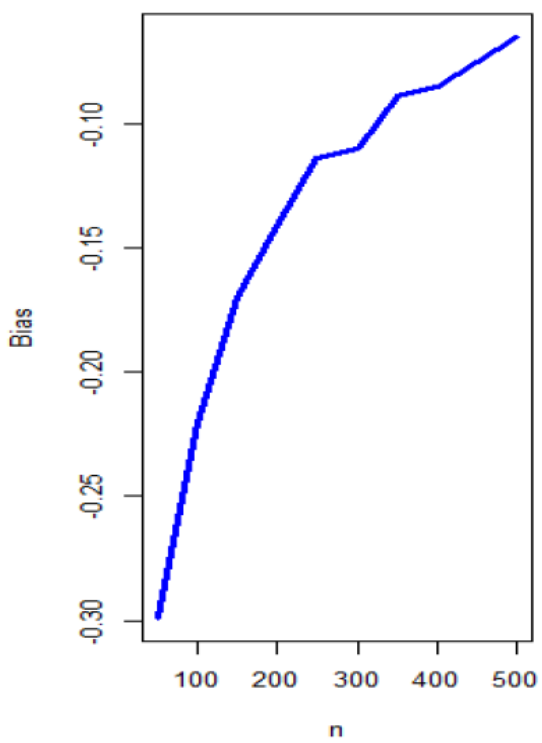

MSE of the parameter $\beta$

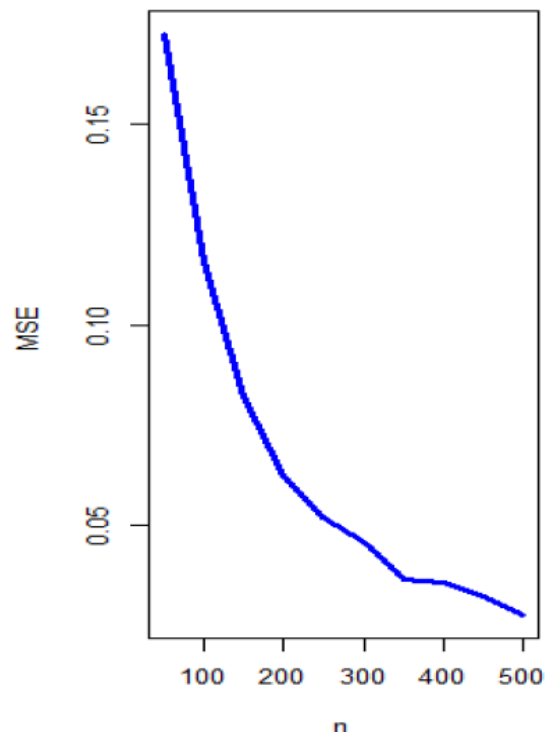

Figure 13. biases and mean squared errors for the parameter $\beta$. 


\section{Applications}

In this section, we provide two real life applications to two real data sets to illustrate the importance and flexibility of the OBP model. We compare the fit of the OBP with some well-known competitive models (see Table 7)

Table 7: Competitive models.

\begin{tabular}{llll}
\hline \hline N. & Model & Abbreviation & Author \\
\hline \hline 1 & Pareto type II & P & Lomax [41] \\
2 & Exponentiated P & ExpP & Gupta et al. [30] \\
3 & Kumaraswamy P & KumP & Lemonte and Cordeiro [40] \\
4 & Macdonald P & McP & Lemonte and Cordeiro [40] \\
5 & Beta P & BP & Lemonte and Cordeiro [40] \\
6 & Gamma P & GamP & Cordeiro et al. [23] \\
7 & Transmuted Topp-Leone P & TTLP & Yousof et al. [68] \\
8 & Reduced TTLP & RTTLP & Yousof et al. [68] \\
9 & Odd log-logistic P & OLLP & Altun et al. [12] \\
10 & Reduced OLLP & ROLLP & Altun et al. [12] \\
11 & Reduced Burr-Hatke P & RBHP & Yousof et al. [73] \\
12 & Reduced OBP & ROBP & New \\
13 & Proportional reversed hazard rate P & PRHRP & New \\
14 & Special generalized mixture P & SGMP & Chesneau and Yousof [22] \\
\hline \hline
\end{tabular}

For checking the normality, the Quantile-Quantile (Q-Q) plot is sketched. For exploring the HRF for real data, the total time test (TTT) plot is provided. For exploring the initial shape of real data nonparametrically, kernel density estimation (KDE) is provided. Figures 12 and 13 give the box plot, normal Q-Q plot, TTT plot and nonparametric KDE for data set I and data set II respectively. Based on Figures 12(a) and 13(a), we note that no extreme values were found in the two real life data sets. Based on Figures 12(b) and 13(b), we note that the normality is nearly exists. Based on Figures 12(c) and 13(c), we note that the HRF is "increasing" for the two real life data sets. Figures 12(d) and 13(d) show nonparametric KDE for exploring the data.

We estimate the unknown parameters of each model by maximum likelihood using "L-BFGS-B" method and the goodness-of-fit statistics Akaike information criterion (AIC), Bayesian IC (BIC), Consistent AIC (CAIC), Hannan-Quinn IC (HQIC), Anderson-Darling $\left(A^{*}\right)$ and Cramér-von Mises $\left(W^{*}\right)$ are used to compare the five models. In general, the smaller the values of these statistics, the better the fit to the data. The required computations are obtained by using the "maxLik" and "goftest" sub-routines in R-software. For failure times data: the analysis results of are listed in Tables 8 and 9. Table 8 gives the MLEs and standard errors (SEs) for failure times data. Table 9 gives the $-\hat{\ell}$ and goodness-of-fits statistics for failure times data. For service times data: the analysis results of are listed in Tables 10 and 11. Table 10 gives the MLEs and SEs for service times data. Table 11 give the $-\hat{\ell}$ and goodness-of-fits statistics for the service times data. Based on Tables 9 and 11, we note that the OBP model gives the lowest values for the AIC, CAIC, BIC, HQIC, $A^{*}$ and $W^{*}$ among all fitted models. Hence, it could be chosen as the best model under these criteria.

Figure 14 gives the estimated PDF (EPDF) plot, estimated CDF (ECDF) plot, probability-probability (P-P) plot and estimated HRF (EHRF) plot for data set I. Figure 15 gives the EPDF plot, ECDF plot, P-P plot and EHRF plot for data set II. From Figures 14 and 15, it is noted that the OBP provides a very adequate fits to the empirical functions. 


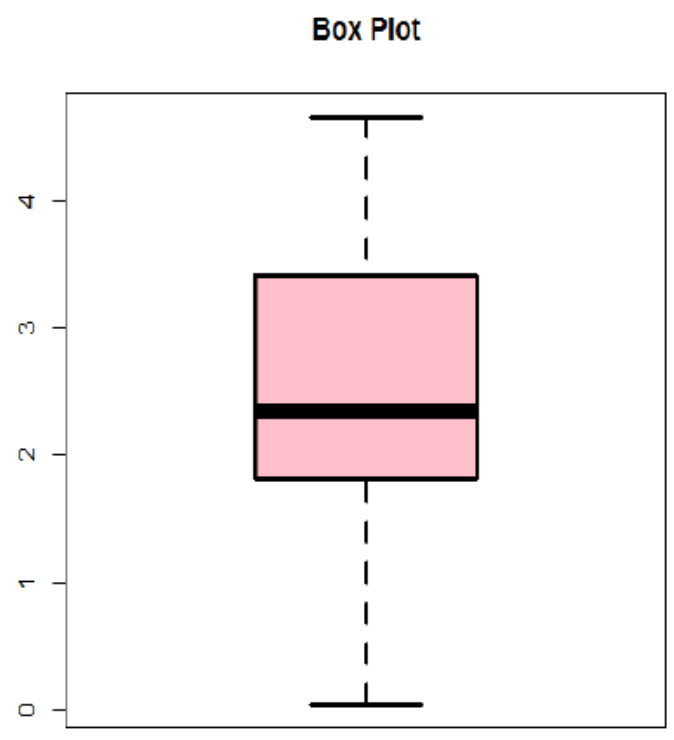

(a)

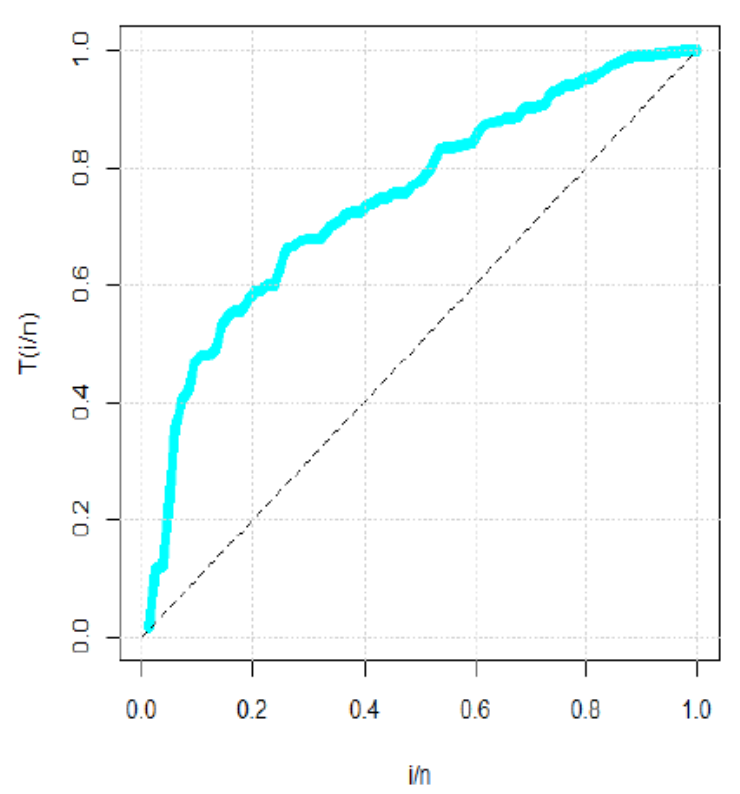

(c)

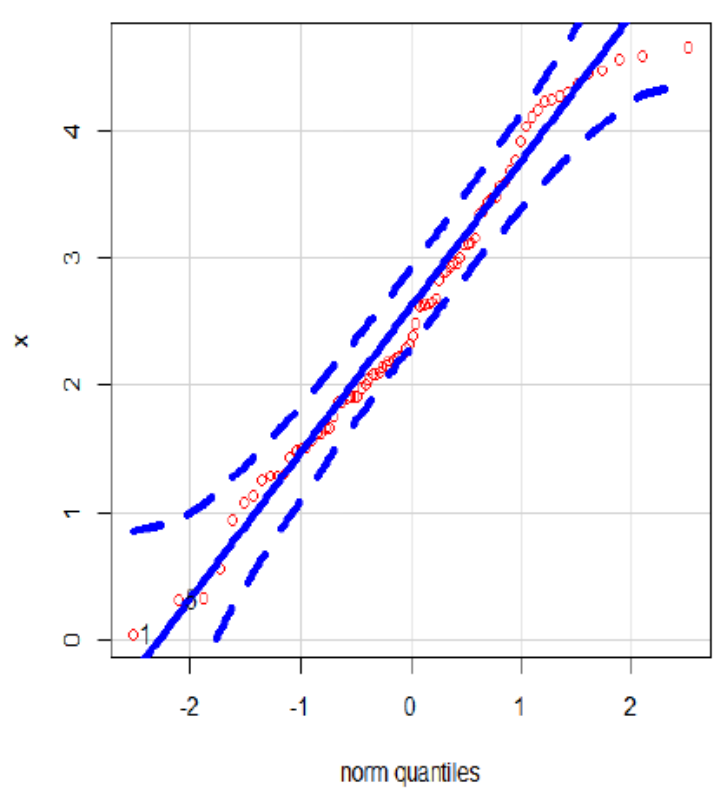

(b)

Kernel Density Estimation from the Raw Data

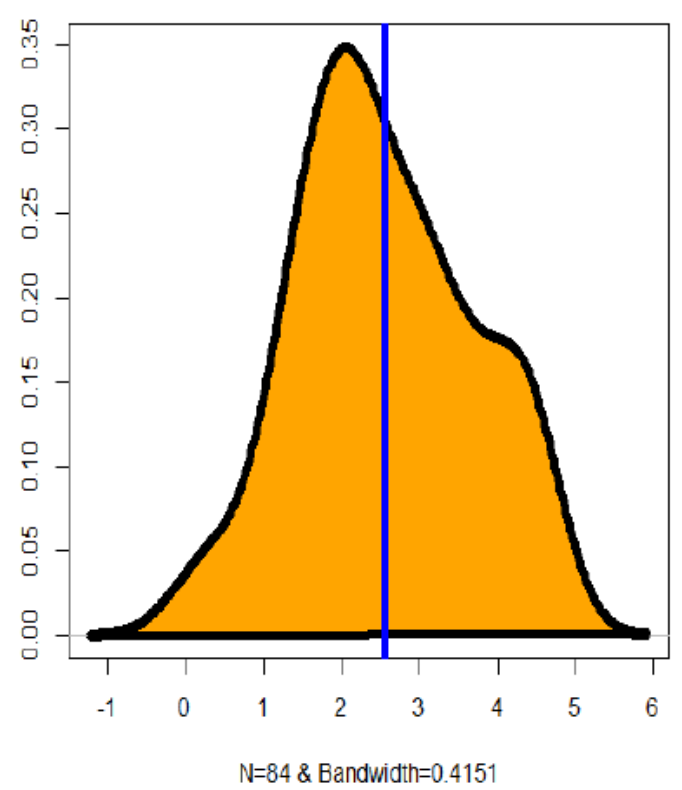

(d)

Figure 14. Box plot, normal Q-Q plot, TTT plot and nonparametric KDE for data set I. 


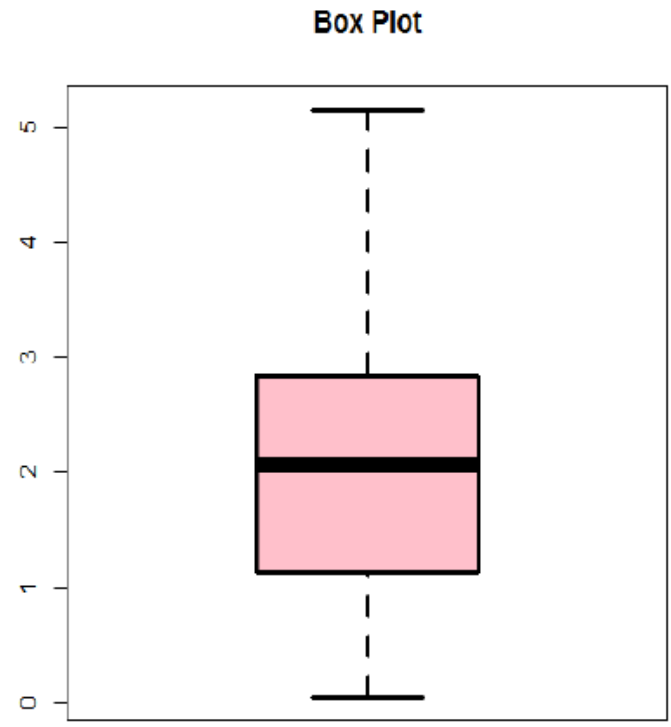

(a)

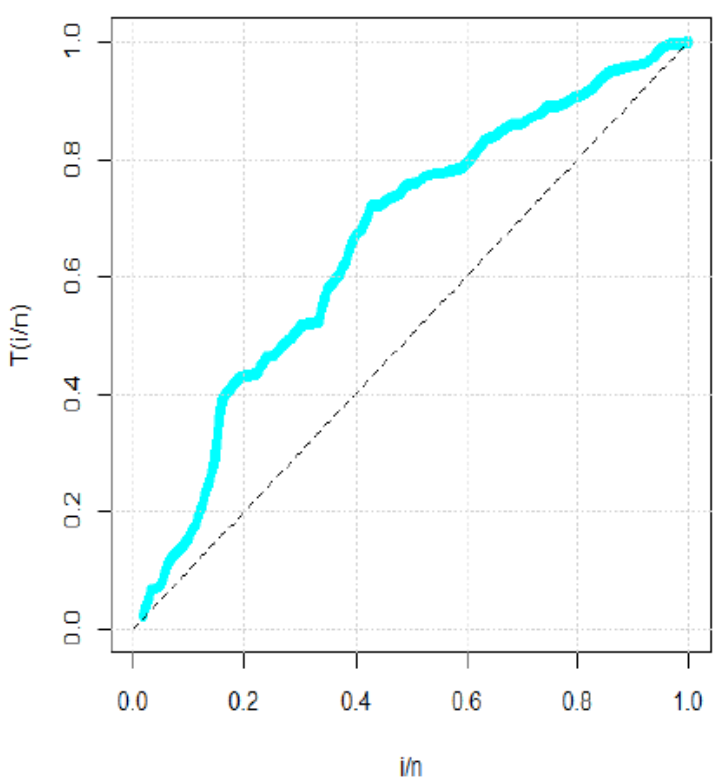

(c)

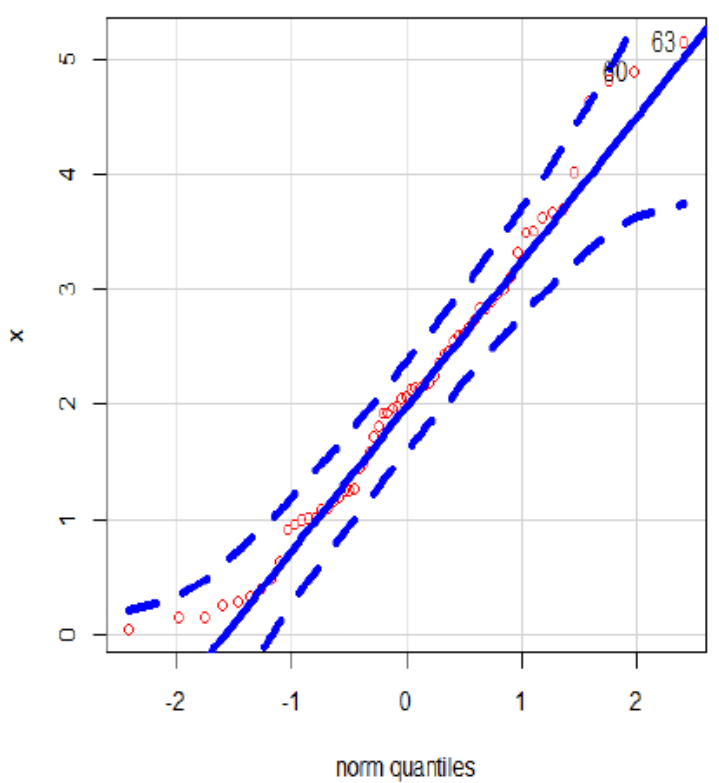

(b)

Kernel Density Estimation from the Raw Data

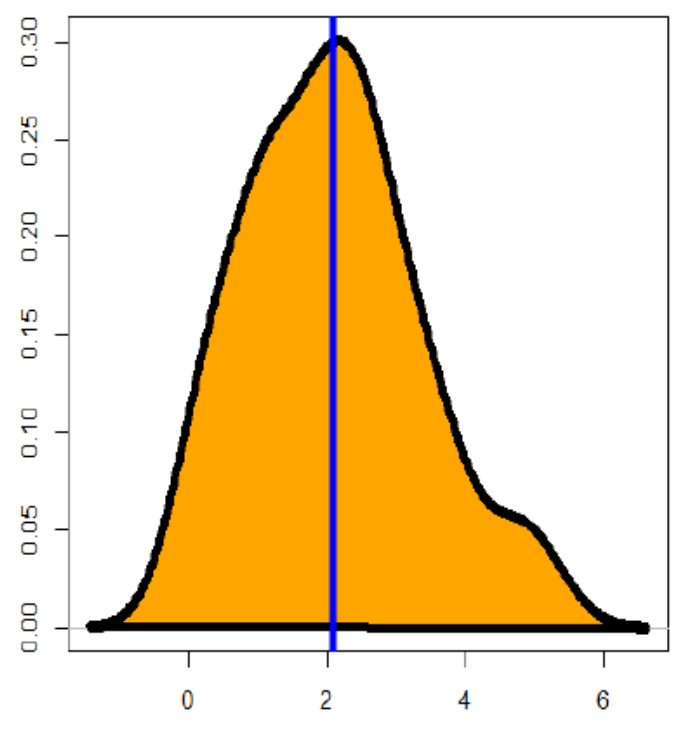

$\mathrm{N}=63$ \& Bandwidth $=0.4894$

(d)

Figure 15. Box plot, normal Q-Q plot, TTT plot and nonparametric KDE for data set II. 
Table 8: MLEs and SEs for failure times data.

\begin{tabular}{|c|c|c|c|c|c|}
\hline \multirow{2}{*}{$\begin{array}{c}\text { Model } \\
\mathbf{O B P}(v, \theta, \alpha, \beta)\end{array}$} & \multicolumn{4}{|c|}{ Estimates } & \\
\hline & $\begin{array}{l}2.27526 \\
(7.2387)\end{array}$ & $\begin{array}{l}57.35316 \\
(21.9898)\end{array}$ & $\begin{array}{l}6.576097 \\
(9.18131)\end{array}$ & $\begin{array}{l}119.4169 \\
(277.895)\end{array}$ & \\
\hline $\operatorname{McP}(v, \theta, c, \alpha, \beta)$ & $\begin{array}{l}2.1875 \\
(0.5211)\end{array}$ & $\begin{array}{l}119.1751 \\
(140.297)\end{array}$ & $\begin{array}{l}12.4171 \\
(20.845)\end{array}$ & $\begin{array}{l}19.9243 \\
(38.9601)\end{array}$ & $\begin{array}{l}75.6606 \\
(147.24)\end{array}$ \\
\hline $\operatorname{TTLP}(v, \theta, \alpha, \beta)$ & $\begin{array}{l}-0.80750 \\
(0.13960)\end{array}$ & $\begin{array}{l}2.47663 \\
(0.54176)\end{array}$ & $\begin{array}{l}(15608.2) \\
(1602.37)\end{array}$ & $\begin{array}{l}(38628.3) \\
(123.936)\end{array}$ & \\
\hline $\operatorname{KumP}(v, \theta, \alpha, \beta)$ & $\begin{array}{l}2.6150 \\
(0.3822)\end{array}$ & $\begin{array}{l}100.2756 \\
(120.486)\end{array}$ & $\begin{array}{l}5.27710 \\
(9.8116)\end{array}$ & $\begin{array}{l}78.6774 \\
(186.005)\end{array}$ & \\
\hline $\mathrm{BP}(v, \theta, \alpha, \beta)$ & $\begin{array}{l}3.60360 \\
(0.6187)\end{array}$ & $\begin{array}{l}33.63870 \\
(63.7145)\end{array}$ & $\begin{array}{l}4.830700 \\
(9.23820)\end{array}$ & $\begin{array}{l}118.8374 \\
(428.927)\end{array}$ & \\
\hline $\operatorname{PRHRP}(\theta, \alpha, \beta)$ & $\begin{array}{l}3.73 \times 10^{6} \\
1.01 \times 10^{6}\end{array}$ & $\begin{array}{l}4.707 \times 10^{-1} \\
(0.00001)\end{array}$ & $\begin{array}{l}4.49 \times 10^{6} \\
37.14684\end{array}$ & & \\
\hline $\operatorname{RTTLP}(v, \theta, \alpha)$ & $\begin{array}{l}-0.84732 \\
(0.10010)\end{array}$ & $\begin{array}{l}5.52057 \\
(1.18479)\end{array}$ & $\begin{array}{l}1.15678 \\
(0.09588)\end{array}$ & & \\
\hline $\operatorname{SGMP}(v, \alpha, \beta)$ & $\begin{array}{l}-1.04 \times 10^{-1} \\
(0.1223)\end{array}$ & $\begin{array}{l}9.83 \times 10^{6} \\
(4843.3)\end{array}$ & $\begin{array}{l}1.18 \times 10^{7} \\
(501.04)\end{array}$ & & \\
\hline $\operatorname{ROBP}(v, \theta, \alpha)$ & $\begin{array}{l}3.54793 \\
(0.3141)\end{array}$ & $\begin{array}{l}30.63743 \\
(55.8404)\end{array}$ & $\begin{array}{l}0.24295 \\
(0.1026)\end{array}$ & & \\
\hline $\operatorname{OLLP}(v, \alpha, \beta)$ & $\begin{array}{l}2.32636 \\
\left(2.14 \times 10^{-1}\right)\end{array}$ & $\begin{array}{l}\left(7.17 \times 10^{5}\right) \\
\left(1.19 \times 10^{4}\right)\end{array}$ & $\begin{array}{l}\left.2.34 \times 10^{6}\right) \\
\left(2.61 \times 10^{1}\right)\end{array}$ & & \\
\hline $\operatorname{GamP}(v, \alpha, \beta)$ & $\begin{array}{l}3.58760 \\
(0.5133)\end{array}$ & $\begin{array}{l}52001.49 \\
(7955.00)\end{array}$ & $\begin{array}{l}37029.66 \\
(81.1644)\end{array}$ & & \\
\hline $\operatorname{ExpP}(v, \alpha, \beta)$ & $\begin{array}{l}3.62610 \\
(0.6236)\end{array}$ & $\begin{array}{l}20074.51 \\
(2041.83)\end{array}$ & $\begin{array}{l}26257.68 \\
(99.7417)\end{array}$ & & \\
\hline $\operatorname{ROLLP}(v, \alpha)$ & $\begin{array}{l}3.890564 \\
(0.36524)\end{array}$ & $\begin{array}{l}0.57316 \\
(0.01946)\end{array}$ & & & \\
\hline $\operatorname{RBHP}(\alpha, \beta)$ & $\begin{array}{l}10801754 \\
(983309)\end{array}$ & $\begin{array}{l}51367189 \\
(232312)\end{array}$ & & & \\
\hline $\mathrm{P}(\alpha, \beta)$ & $\begin{array}{l}51425.35 \\
(5933.49)\end{array}$ & $\begin{array}{l}131789.8 \\
(296.119)\end{array}$ & & & \\
\hline
\end{tabular}


Table 9: $-\hat{\ell}$ and goodness-of-fits statistics for failure times data.

\begin{tabular}{cccccccc}
\hline \hline Model & $\hat{\ell}$ & AIC & CAIC & BIC & HQIC & $A^{*}$ & $W^{*}$ \\
\hline \hline OBP & $\mathbf{- 1 2 9 . 5 2 8 7}$ & $\mathbf{2 6 7 . 0 5 7}$ & $\mathbf{2 6 7 . 5 6 2}$ & $\mathbf{2 7 6 . 7 7 9}$ & $\mathbf{2 7 0 . 9 6 4}$ & $\mathbf{0 . 5 6 7 5}$ & $\mathbf{0 . 0 5 8 4}$ \\
McP & -129.8023 & 269.6045 & 270.3640 & 281.8178 & 274.5170 & 0.6672 & 0.0858 \\
KumP & -132.4048 & 272.8096 & 273.3096 & 282.5802 & 276.7396 & 0.6645 & 0.0658 \\
ROBP & -134.3584 & 274.7169 & 275.0169 & 282.0093 & 277.6484 & 0.9439 & 0.1004 \\
OLLP & -134.4235 & 274.8470 & 275.1470 & 282.1394 & 277.7785 & 0.9407 & 0.1009 \\
TTLP & -135.5700 & 279.1400 & 279.6464 & 288.8633 & 283.0487 & 1.1257 & 0.1270 \\
GamP & -138.4042 & 282.8083 & 283.1046 & 290.1363 & 285.7559 & 1.3666 & 0.1618 \\
BP & -138.7177 & 285.4354 & 285.9354 & 295.2060 & 289.3654 & 1.4084 & 0.1680 \\
ExpP & -141.3997 & 288.7994 & 289.0957 & 296.1273 & 291.7469 & 1.7435 & 0.2194 \\
ROLLP & -142.8452 & 289.6904 & 289.8385 & 294.5520 & 291.6447 & 1.9566 & 0.2554 \\
SGMP & -143.0874 & 292.1747 & 292.4747 & 299.4672 & 295.1062 & 1.3467 & 0.1578 \\
RTTLP & -153.9809 & 313.9618 & 314.2618 & 321.2542 & 316.8933 & 3.7527 & 0.5592 \\
PRHRP & -162.8770 & 331.7540 & 332.0540 & 339.0464 & 334.6855 & 1.3672 & 0.1609 \\
P & -164.9884 & 333.9767 & 334.1230 & 338.8620 & 335.9417 & 1.3976 & 0.1665 \\
RBHP & -168.6040 & 341.2081 & 341.3562 & 346.0697 & 343.1624 & 1.6711 & 0.2069 \\
\hline \hline
\end{tabular}

Table 10: MLEs and SEs for service times data.

\begin{tabular}{|c|c|c|c|c|}
\hline \multirow{2}{*}{$\begin{array}{c}\text { Model } \\
\text { OBP }(v, \theta, \alpha, \beta)\end{array}$} & \multicolumn{4}{|c|}{ Estimates } \\
\hline & $\begin{array}{l}1.62783 \\
(0.16217)\end{array}$ & $\begin{array}{l}318.092 \\
(76.8271)\end{array}$ & $\begin{array}{l}2.21029 \\
(4.5955)\end{array}$ & $\begin{array}{l}177.4062 \\
(522.549)\end{array}$ \\
\hline $\operatorname{KumP}(v, \theta, \alpha, \beta)$ & $\begin{array}{l}1.6691 \\
(0.2570)\end{array}$ & $\begin{array}{l}60.5673 \\
(86.0131)\end{array}$ & $\begin{array}{l}2.56490 \\
(4.7589)\end{array}$ & $\begin{array}{l}65.06400 \\
(177.592)\end{array}$ \\
\hline $\mathrm{BP}(v, \theta, \alpha, \beta)$ & $\begin{array}{l}1.9218 \\
(0.3184)\end{array}$ & $\begin{array}{l}31.2594 \\
(316.841)\end{array}$ & $\begin{array}{l}4.9684 \\
(50.528)\end{array}$ & $\begin{array}{l}169.5719 \\
(339.207)\end{array}$ \\
\hline $\operatorname{TTLP}(v, \theta, \alpha, \beta)$ & $\begin{array}{l}(-0.6070) \\
(0.21371)\end{array}$ & $\begin{array}{l}1.785780 \\
(0.41522)\end{array}$ & $\begin{array}{l}2123.391 \\
(163.915)\end{array}$ & $\begin{array}{l}4822.789 \\
(200.009)\end{array}$ \\
\hline $\operatorname{PRHRP}(\theta, \alpha, \beta)$ & $\begin{array}{l}1.59 \times 10^{6} \\
2.01 \times 10^{3}\end{array}$ & $\begin{array}{l}3.93 \times 10^{-1} \\
0.0004 \times 10^{-1}\end{array}$ & $\begin{array}{l}1.30 \times 10^{6} \\
0.95 \times 10^{6}\end{array}$ & \\
\hline $\operatorname{RTTLP}(v, \theta, \alpha)$ & $\begin{array}{l}-0.67145 \\
(0.18746)\end{array}$ & $\begin{array}{l}2.74496 \\
(0.6696)\end{array}$ & $\begin{array}{l}1.01238 \\
(0.11405)\end{array}$ & \\
\hline $\operatorname{SGMP}(v, \alpha, \beta)$ & $\begin{array}{l}-1.04 \times 10^{-1} \\
\left(4.1 \times 10^{-10}\right)\end{array}$ & $\begin{array}{l}6.45 \times 10^{6} \\
\left(3.21 \times 10^{6}\right)\end{array}$ & $\begin{array}{l}6.33 \times 10^{6} \\
(3.8573)\end{array}$ & \\
\hline $\operatorname{ROBP}(v, \theta, \alpha)$ & $\begin{array}{l}2.35836 \\
(0.2413)\end{array}$ & $\begin{array}{l}23.13999 \\
(41.18194)\end{array}$ & $\begin{array}{l}0.20245 \\
(0.1325)\end{array}$ & \\
\hline $\operatorname{OLLP}(v, \alpha, \beta)$ & $\begin{array}{l}1.66419 \\
\left(1.79 \times 10^{-1}\right)\end{array}$ & $\begin{array}{l}6.340 \times 10^{5} \\
\left(1.68 \times 10^{4}\right)\end{array}$ & $\begin{array}{l}2.01 \times 10^{6} \\
7.22 \times 10^{6}\end{array}$ & \\
\hline $\operatorname{GamP}(v, \alpha, \beta)$ & $\begin{array}{l}1.9073 \\
(0.3213)\end{array}$ & $\begin{array}{l}35842.433 \\
(6945.074)\end{array}$ & $\begin{array}{l}39197.57 \\
(151.653)\end{array}$ & \\
\hline $\operatorname{ExpP}(v, \alpha, \beta)$ & $\begin{array}{l}1.9145 \\
(0.3482)\end{array}$ & $\begin{array}{l}22971.154 \\
(3209.533)\end{array}$ & $\begin{array}{l}32881.99 \\
(162.230)\end{array}$ & \\
\hline $\operatorname{ROLLP}(v, \alpha)$ & $\begin{array}{l}2.37233 \\
(0.26825)\end{array}$ & $\begin{array}{l}0.69109 \\
(0.04488)\end{array}$ & & \\
\hline $\operatorname{RBHP}(\alpha, \beta)$ & $\begin{array}{l}14055522 \\
(422.005)\end{array}$ & $\begin{array}{l}53203423 \\
(28.52323)\end{array}$ & & \\
\hline $\mathrm{P}(\alpha, \beta)$ & $\begin{array}{l}99269.78 \\
(11863.5)\end{array}$ & $\begin{array}{l}207019.37 \\
(301.2366)\end{array}$ & & \\
\hline
\end{tabular}



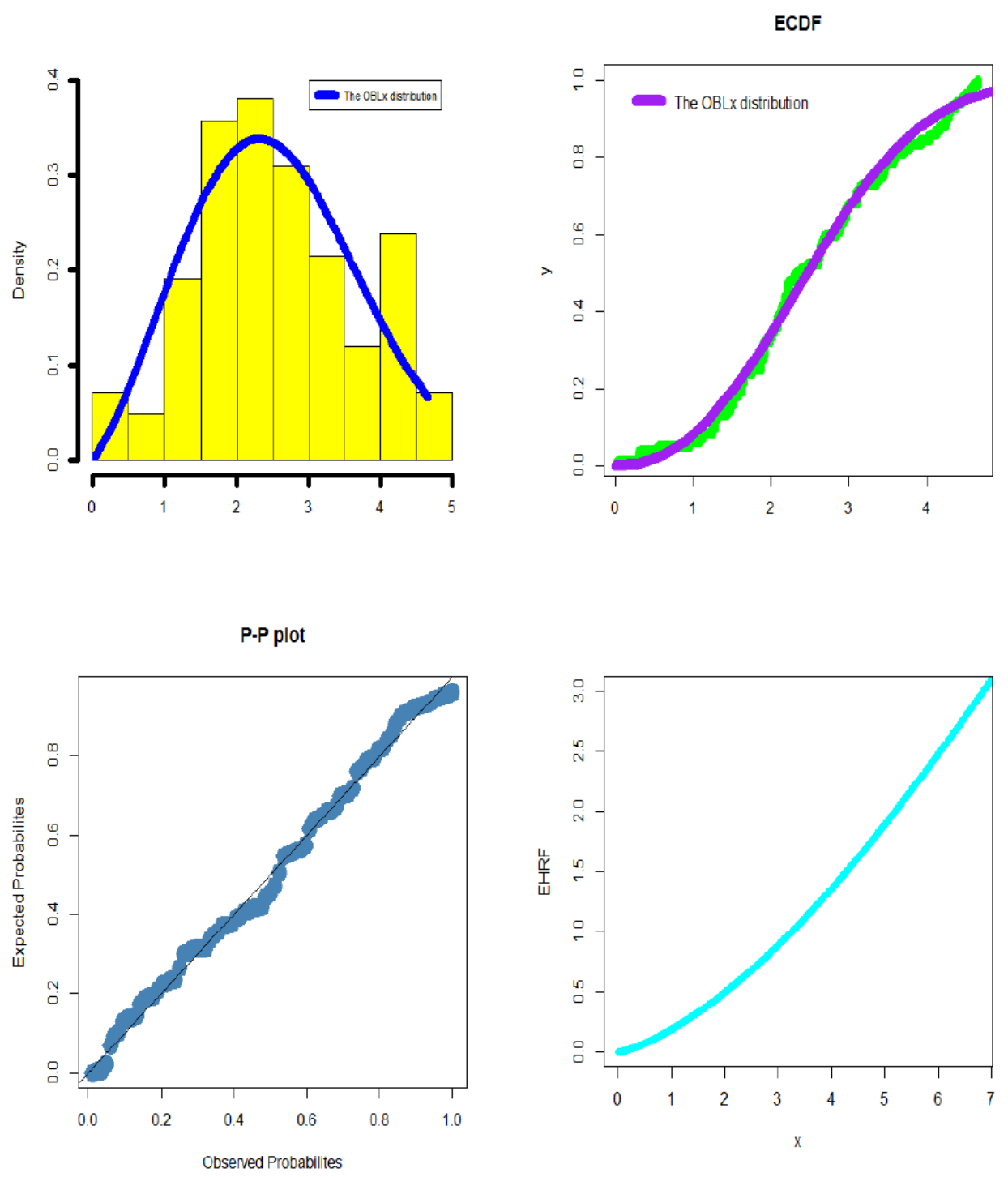

Figure 16. EPDF, ECDF, P-P plot and EHRF for data set I. 
Table 11: $-\hat{\ell}$ and goodness-of-fits statistics for the service times data.

\begin{tabular}{cccccccc}
\hline \hline Model & $\hat{\ell}$ & AIC & CAIC & BIC & HQIC & $A^{*}$ & $W^{*}$ \\
\hline \hline OBP & $-\mathbf{1 0 0 . 0 1 8 3}$ & $\mathbf{2 0 8 . 0 3 7}$ & $\mathbf{2 0 8 . 7 2 6}$ & $\mathbf{2 1 6 . 6 0 9}$ & $\mathbf{2 1 1 . 4 0 8}$ & $\mathbf{0 . 5 7 2 8}$ & $\mathbf{0 . 0 9 4 4}$ \\
KumP & -100.8676 & 209.7353 & 210.4249 & 218.3078 & 213.1069 & 0.7391 & 0.1219 \\
TTLP & -102.4498 & 212.8996 & 213.5893 & 221.4722 & 216.2713 & 0.9431 & 0.1554 \\
GamP & -102.8332 & 211.6663 & 212.0730 & 218.0958 & 214.1951 & 1.1120 & 0.1836 \\
SGMP & -102.8940 & 211.7881 & 212.1949 & 218.2175 & 214.3168 & 1.1134 & 0.1839 \\
BP & -102.9611 & 213.9223 & 214.6119 & 222.4948 & 217.2939 & 1.1336 & 0.1872 \\
ExpP & -103.5498 & 213.0995 & 213.5063 & 219.5289 & 215.6282 & 1.2331 & 0.2037 \\
ROBP & -104.4258 & 214.8517 & 215.2584 & 221.2811 & 217.3804 & 1.2825 & 0.2115 \\
OLLP & -104.9041 & 215.8082 & 216.2150 & 222.2376 & 218.3369 & 0.9424 & 0.1545 \\
PRHRP & -109.2986 & 224.5973 & 225.004 & 231.0267 & 227.126 & 1.1264 & 0.1861 \\
P & -109.2988 & 222.5976 & 222.7976 & 226.8839 & 224.2834 & 1.1265 & 0.1861 \\
ROLLP & -110.7287 & 225.4573 & 225.6573 & 229.7436 & 227.1431 & 2.3472 & 0.3908 \\
RTTLP & -112.1855 & 230.3710 & 230.7778 & 236.8004 & 232.8997 & 2.6875 & 0.4532 \\
RBHP & -112.6005 & 229.2011 & 229.4011 & 233.4873 & 230.8869 & 1.3984 & 0.2316 \\
\hline \hline
\end{tabular}

\section{Conclusions}

A new lifetime model called the odd Burr Pareto type II (OBP) model is introduced and studied. The major motivation for the practicality of the OBP model is based on the wider importance of the standard Pareto type II model. TheOBP density can be "right skewed" with heavy tail shape. The OBP failure rate can be "J-shape", "decreasing" and "upside down (or increasing-constant-decreasing)". The new OBP density can be expressed as as a mixture of the exponentiated Pareto type II model. The skewness of the OBP distribution can range in the interval $(-69216.87,98983.16)$. The spread for the kurtosis of the OBP model is ranging from 74.17124 to $\infty$. The index of dispersion for the OBP model can be in $(0,1)$ an dalso $>1$ so it may be used as an "under-dispersed" and "over-dispersed" model. Bayesian and non-Bayesian estimation methods are considered. We assessed the performance of all methods via simulation study. Bayesian and many non-Bayesian estimation methods (such as uch as the maximum likelihood estimation method, ordinary least square estimation method, weighted least square estimationmethod and the Kolmogorov estimation method) are compared in modeling real data via two applications. In modeling real data, the maximum likelihood method is the best estimation method. So, we used it in comparing competitive models. Before using the the maximum likelihood method, we performed simulation experiments to assess the finite sample behavior of it using the biases and mean squared errors. The OBP model could be chosen as the best model among Pareto type II, exponentiated Pareto type II, Kumaraswamy Pareto type II, Macdonald Pareto type II, beta Pareto type II, gamma Pareto type II, odd log-logistic Pareto type II, reduced odd log-logistic Pareto type II, reduced Burr-Hatke Pareto type II, reduced OBP and special generalized mixture Pareto type II distribution in modeling the "failure times" and the "service times" data sets.

As a future potential work we may consider the modified Nikulin-Rao-Robson goodness-of-fit test for distributional validation as presented by Abouelmagd et al. [1], Abouelmagd et al. [2], Ibrahim et al. [39], brahim et al. [37], Goual et al. [28] and Goual et al. [29]. We may consider the modified Bagdonavičius-Nikulin Goodnessof-fit test for censored validation as presented by Mansour et al. ([53], [54], [56], [57], [52] and [55]), Yousof et al. [67] and Salah et al. [61]. Finally, we may characterize the OBP model using some characterizations theorems such as conditional expectation, truncated moment, hazard rate function, Mills ratio, certain functions of the random variable, first order statistic and conditional expectation of the record values as presented by Hamedani et al. ([46],[47] and [48]), Khalil et al. [49], Altun et al. ([13], [14], [15] and [17]), Alizadeh et al. ([7], [8] and [9]), Yousof et al. ([69], [70], [71], [72]), Altun et al. ([11] and [16]) and Ibrahim et al. [38]. 

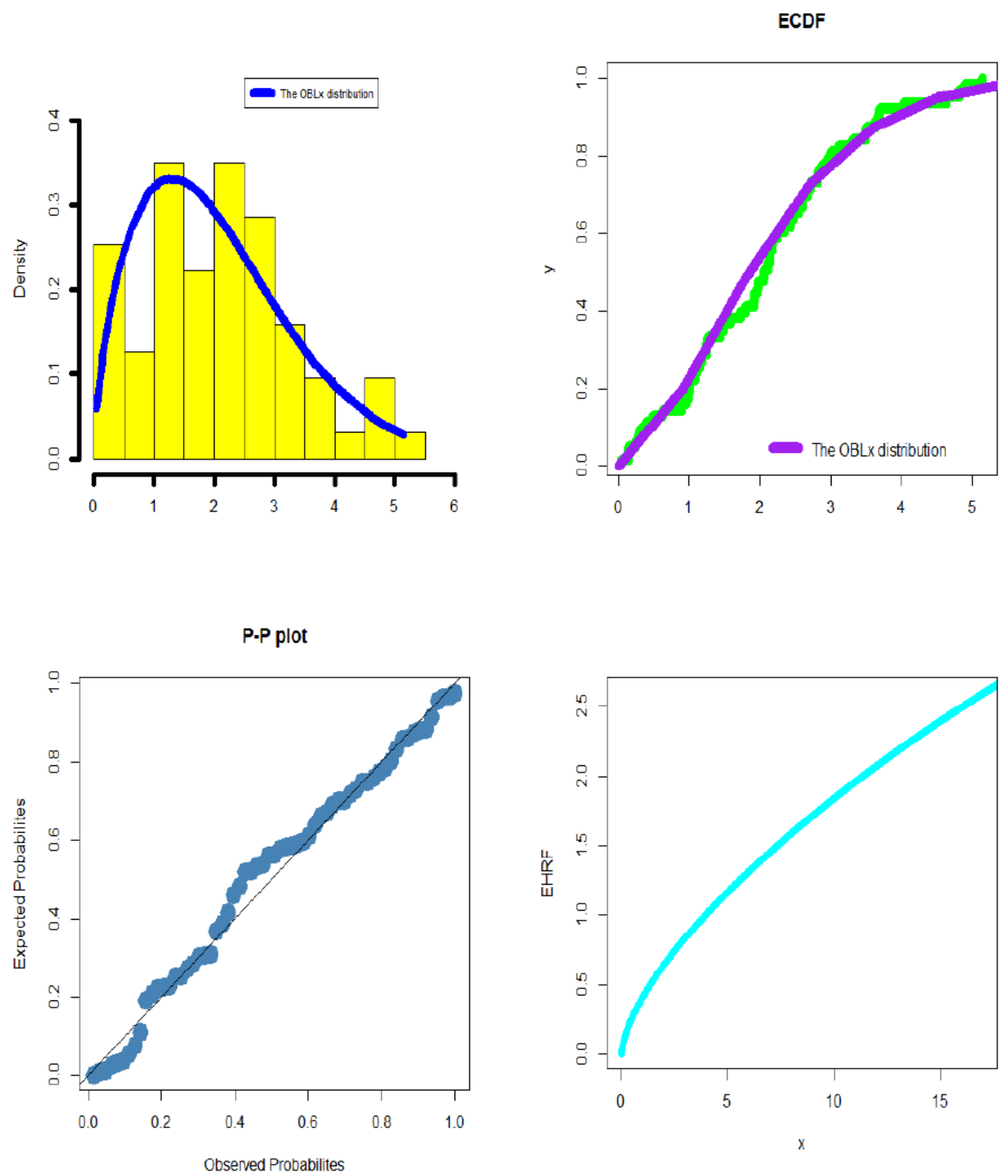

Figure 17. EPDF, ECDF, P-P plot and EHRF for data set II.

\section{REFERENCES}

1. Abouelmagd, T. H. M., Hamed, M. S., Hamedani, G. G., Ali, M. M., Goual, H., Korkmaz, M. C. and Yousof, H. M. (2019a). The zero truncated Poisson Burr X family of distributions with properties, characterizations, applications, and validation test. Journal of Nonlinear Sciences and Applications, 12(5), 314-336.

2. Abouelmagd, T. H. M., Hamed, M. S., Handique, L., Goual, H., Ali, M. M., Yousof, H. M. and Korkmaz, M. C. (2019b). A new class of distributions based on the zero truncated Poisson distribution with properties and applications. Journal of Nonlinear Sciences \& Applications (JNSA), 12(3), 152-164.

3. Al-babtain, A. A., Elbatal, I., \& Yousof, H. M. (2020). A new flexible three-parameter model: properties, clayton copula, and modeling real data. Symmetry, 12(3), 440. 
4. Ali, M. M., Yousof, H. M. and Ibrahim, M. (2021a). A new version of the generalized Rayleigh distribution with copula, properties, applications and different methods of estimation. Optimal Decision Making in Operations Research \& Statistics: Methodologies and Applications. To appear.

5. Ali, M. M., Ibrahim, M. and Yousof, H. M. (2021b). Expanding the Burr X model: properties, copula, real data modeling and different methods of estimation. Optimal Decision Making in Operations Research \& Statistics: Methodologies and Applications. To appear.

6. Alizadeh, M., Cordeiro, G. M., Nascimento, A. D., Lima, M. D. C. S. and Ortega, E. M. (2017a). Odd-Burr generalized family of distributions with some applications. Journal of statistical computation and simulation, 87(2), 367-389.

7. Alizadeh, M., Ghosh, I., Yousof, H. M., Rasekhi, M. and Hamedani, G. G. (2017b). The generalized odd generalized exponential family of distributions: properties, characterizations and applications. Journal of Data Science, 15(3), 443-465.

8. Alizadeh, M., Jamal, F., Yousof, H. M., Khanahmadi, M. and Hamedani, G. G. (2020). Flexible Weibull generated family of distributions: characterizations, mathematical properties and applications. University Politehnica of Bucharest Scientific BulletinSeries A-Applied Mathematics and Physics, 82(1), 145-150.

9. Alizadeh, M., Rasekhi, M., Yousof, H. M., Ramires, T. G. and Hamedani, G. G. (2018). Extended exponentiated Nadarajah-Haghighi model: Mathematical properties, characterizations and applications. Studia Scientiarum Mathematicarum Hungarica, 55(4), 498522.

10. Atkinson, A. B. and Harrison, A. J. (1978). Distribution of Personal Wealth in Britain (Cambridge University Press, Cambridge).

11. Altun, E. Alizadeh, M., Yousof, H. M., Rasekhi, M. and Hamedani, G. G. (2021a). A New Type II Half Logistic-G family of Distributions with Properties, Regression Models, Reliability Systems and Applications. Applications and Applied Mathematics: An International Journal. forthcoming.

12. Altun, E., Yousof, H. M. and Hamedani G. G. (2018a). A flexible extension of generalized half-normal distribution: characterizations and regression models. International Journal of Applied Mathematics and Statistics, 57(3), 27-49.

13. Altun, E., Yousof, H. M. and Hamedani G. G. (2018b). A new flexible extension of the generalized half-normal lifetime model with characterizations and regression modeling. Bulletin of Computational Applied Mathematics, 6(1), 83-115.

14. Altun, E., Yousof, H. M. and Hamedani, G. G. (2018c). A new generalization of generalized half-normal distribution: properties and regression models. Journal of Statistical Distributions and Applications, 5(1), 7.

15. Altun, E., Yousof, H. M. and Hamedani, G. G. (2018d). A new log-location regression model with influence diagnostics and residual analysis. Facta Universitatis, Series: Mathematics and Informatics, 33(3), 417-449.

16. Altun, E., Yousof, H. M. and Hamedani, G. G. (2021b). The Gudermannian generated family of distributions with characterizations, regression models and applications. Studia Scientiarum Mathematicarum Hungarica, forthcoming.

17. Altun, E., Yousof, H. M., Chakraborty, S. and Handique, L. (2018e). Zografos-Balakrishnan Burr XII distribution: regression modeling and applications. International Journal of Mathematics and Statistics, 19(3), 46-70.

18. Aryal, G. R., Ortega, E. M., Hamedani, G. G. and Yousof, H. M. (2017). The Topp-Leone generated Weibull distribution: regression model, characterizations and applications. International Journal of Statistics and Probability, 6(1), 126-141.

19. Balkema, A.A. and de Hann, L. Residual life at great age, Annals of Probability 2, 972-804, 1974.

20. Bryson, M. C. (1974). Heavy-tailed distribution: properties and tests. Technometrics 16, 161-68.

21. Chahkandi, M. and Ganjali, M. (2009). On some lifetime distributions with decrasing failure rate. Computational Statistics and Data Analysis 53, 4433-4440.

22. Chesneau, C. and Yousof, H. M. (2021). On a special generalized mixture class of probabilitic models. Journal of Nonlinear Modeling and Analysis, 3(1), 71-92.

23. Cordeiro, G. M., Yousof, H. M., Ramires, T. G. and Ortega, E. M. M. (2018). The Burr XII system of densities: properties, regression model and applications. Journal of Statistical Computation and Simulation, 88(3), 432-456.

24. Durbey, S. D. (1970). Compound gamma, beta and F distributions. Metrika 16, 27-31.

25. Elgohari, H. and Yousof, H. M. (2020a). A generalization of Lomax distribution with properties, copula and real data applications. Pakistan Journal of Statistics and Operation Research, 16(4), 697-711. https://doi.org/10.18187/pjsor.v16i4.3260

26. Elgohari, H. and Yousof, H. M. (2020b). New extension of Weibull distribution: copula, mathematical properties and data modeling. Statistics, Optimization \& Information Computing, 8(4), 972-993. https://doi.org/10.19139/soic-2310-5070-1036

27. Gad, A. M., Hamedani, G. G., Salehabadi, S. M. and Yousof, H. M. (2019). The Burr XII-Burr XII distribution: mathematical properties and characterizations. Pakistan Journal of Statistics, 35(3), 229-248.

28. Goual, H., Yousof, H. M. and Ali, M. M. (2019). Validation of the odd Lindley exponentiated exponential by a modified goodness of fit test with applications to censored and complete data. Pakistan Journal of Statistics and Operation Research, 15(3), 745-771.

29. Goual, H., Yousof, H. M. and Ali, M. M. (2020). Lomax inverse Weibull model: properties, applications, and a modified Chi-squared goodness-of-fit test for validation. Journal of Nonlinear Sciences \& Applications (JNSA), 13(6), 330-353.

30. Gupta, R. C., Gupta, P. L. and Gupta, R. D. (1998). Modeling failure time data by Lehman alternatives. Communications in StatisticsTheory and methods, 27(4), 887-904.

31. Corbellini, A., Crosato, L., Ganugi, P and Mazzoli, M. (2007). Fitting Pareto II distributions on firm size: Statistical methodology and economic puzzles. Paper presented at the International Conference on Applied Stochastic Models and Data Analysis, Chania, Crete.

32. Cordeiro, G. M., Ortega, E. M. and Popovic, B. V. (2015). The gamma-Lomax distribution. Journal of Statistical computation and Simulation, 85(2), 305-319.

33. Elbiely, M. M. and Yousof, H. M. (2018). A new extension of the Lomax distribution and its Applications. Journal of Statistics and Applications, 2(1), 18-34.

34. Harris, C.M. (1968). The Pareto distribution as a queue service descipline. Operations Research, 16, 307-313.

35. Hassan, A.S. and Al-Ghamdi, A.S. (2009). Optimum step stress accelerated life testing for Lomax distibution. Journal of Applied Sciences Research, 5, 2153-2164.

36. Ibrahim, M. and Yousof, H. M. (2020). A new generalized Lomax model: statistical properties and applications. Journal of Data Science, $18(1), 190-217$.

37. Ibrahim, M., Altun, E., Goual, H., and Yousof, H. M. (2020). Modified goodness-of-fit type test for censored validation under a new Burr type XII distribution with different methods of estimation and regression modeling. Eurasian Bulletin of Mathematics, 3(3), 
162-182.

38. Ibrahim. M., Handique, L., Chakraborty, S., Butt, N. S. and M. Yousof, H. (2021). A new three-parameter xgamma Fréchet distribution with different methods of estimation and applications. Pakistan Journal of Statistics and Operation Research, 17(1), 291-308.

39. Ibrahim, M., Yadav, A. S., Yousof, H. M., Goual, H. and Hamedani, G. G. (2019). A new extension of Lindley distribution: modified validation test, characterizations and different methods of estimation. Communications for Statistical Applications and Methods, 26(5), 473-495.

40. Lemonte, A. J. and Cordeiro, G. M. (2013). An extended Lomax distribution. Statistics, 47(4), 800-816.

41. Lomax, K.S. (1954). Business failures: Another example of the analysis of failure data. Journal of the American Statistical Association, 49, 847-852.

42. Gleaton, J. U. and Lynch, J.D. (2006). Properties of generalized loglogistic families of lifetime distributions. Journal of Probability and Statistical Science, 4, 51-64.

43. Goual, H. and Yousof, H. M. (2019). Validation of Burr XII inverse Rayleigh model via a modified chi-squared goodness-of-fit test. Journal of Applied Statistics, 47(1), 1-32.

44. Gumbel, E. J. (1961). Bivariate logistic distributions. Journal of the American Statistical Association, 56(294), $335-349$.

45. Gumbel, E. J. (1960) Bivariate exponential distributions. Journ. Amer. Statist. Assoc., 55, 698-707.

46. Hamedani, G. G., Altun, E., Korkmaz, M. C., Yousof, H. M. and Butt, N. S. (2018a). A new extended G family of continuous distributions with mathematical properties, characterizations and regression modeling. Pakistan Journal of Statistics and Operation Research, 737-758.

47. Hamedani, G. G., Rasekhi, M., Najibi, S., Yousof, H. M. and Alizadeh, M. (2019). Type II general exponential class of distributions. Pakistan Journal of Statistics and Operation Research, 15(2), 503-523.

48. Hamedani, G. G., Yousof, H. M., Rasekhi, M., Alizadeh, M. and Najibi, S. M., (2018b). Type I general exponential class of distributions. Pakistan Journal of Statistics and Operation Research, 14(1), 39-55.

49. Khalil, M. G., Hamedani, G. G. and Yousof, H. M. (2019). The Burr X exponentiated Weibull model: characterizations, mathematical properties and applications to failure and survival times data. Pakistan Journal of Statistics and Operation Research, 141-160.

50. Kotz, S. and Johnson, N. L. (1992). Breakthroughs in Statistics: Foundations and basic theory. Springer,Volume 1.

51. Kotz, S. and Nadarajah, S. (2000). Extreme value distributions: theory and applications. Imperial College Press, London.

52. Mansour, M. M., Ibrahim, M., Aidi, K., Shafique Butt, N., Ali, M. M., Yousof, H. M. and Hamed, M. S. (2020a). A new log-logistic lifetime model with mathematical properties, copula, modified goodness-of-fit test for validation and real data modeling. Mathematics, $8(9), 1508$.

53. Mansour, M. M., Butt, N. S., Ansari, S. I., Yousof, H. M., Ali, M. M. and Ibrahim, M. (2020b). A new exponentiated Weibull distribution's extension: copula, mathematical properties and applications. Contributions to Mathematics, 1 (2020) 57-66. DOI: $10.47443 / \mathrm{cm} .2020 .0018$

54. Mansour, M., Korkmaz, M. C., Ali, M. M., Yousof, H. M., Ansari, S. I. and Ibrahim, M. (2020c). A generalization of the exponentiated Weibull model with properties, Copula and application. Eurasian Bulletin of Mathematics, 3(2), 84-102.

55. Mansour, M., Rasekhi, M., Ibrahim, M., Aidi, K., Yousof, H. M. and Elrazik, E. A. (2020d). A new parametric life distribution with modified Bagdonavičius-Nikulin goodness-of-fit test for censored validation, properties, applications, and different estimation methods. Entropy, 22(5), 592.

56. Mansour, M., Yousof, H. M., Shehata, W. A. and Ibrahim, M. (2020e). A new two parameter Burr XII distribution: properties, copula, different estimation methods and modeling acute bone cancer data. Journal of Nonlinear Science and Applications, 13(5), 223-238.

57. Mansour, M. M., Butt, N. S., Yousof, H. M., Ansari, S. I. and Ibrahim, M. (2020f). A generalization of reciprocal exponential model: clayton copula, statistical properties and modeling skewed and symmetric real data sets. Pakistan Journal of Statistics and Operation Research, 16(2), 373-386.

58. Murthy, D.N.P. Xie, M. and Jiang, R. (2004). Weibull Models, Wiley.

59. Pougaza, D. B. and Djafari, M. A. (2011). Maximum entropies copulas. Proceedings of the 30th international workshop on Bayesian inference and maximum Entropy methods in Science and Engineering, 329-336.

60. Rodriguez-Lallena, J. A. and Ubeda-Flores, M. (2004). A new class of bivariate copulas. Statistics and Probability Letters, 66, 315-25.

61. Salah, M. M., El-Morshedy, M., Eliwa, M. S. and Yousof, H. M. (2020). Expanded Fréchet model: mathematical properties, copula, different estimation methods, applications and validation testing. Mathematics, 8(11), 1949.

62. Tadikamalla, P. R. (1980). A look at the Burr and realted distributions. International Statistical Review 48, 337-344.

63. Yadav, A. S., Altun, E. and Yousof, H. M. (2019). Burr-Hatke exponential distribution: a decreasing failure rate model, statistical inference and applications. Annals of Data Science, 1-20.

64. Tahir, M. H., Cordeiro, G. M., Mansoor, M. and Zubair, M. (2015). The Weibull-Lomax distribution: properties and applications. Hacettepe Journal of Mathematics and Statistics, 44(2), 461-480.

65. Yadav, A. S., Goual, H., Alotaibi, R. M., Ali, M. M. and Yousof, H. M. (2020). Validation of the Topp-Leone-Lomax model via a modified Nikulin-Rao-Robson goodness-of-fit test with different methods of estimation. Symmetry, 12(1), 57.

66. Yousof, H. M., Ahsanullah, M. and Khalil, M. G. (2019a). A new zero-truncated version of the Poisson Burr XII distribution: characterizations and properties. Journal of Statistical Theory and Applications, 18(1), 1-11.

67. Yousof, H. M., Ali, M. M., Goual, H. and Ibrahim. M. (2021). A new reciprocal Rayleigh extension: properties, copulas, different methods of estimation and modified right censored test for validation. Statistics in Transition New Series, forthcoming.

68. Yousof, H. M., Alizadeh, M., Jahanshahiand, S. M. A., Ramires, T. G., Ghosh, I. and Hamedani, G. G. (2017). The transmuted Topp-Leone G family of distributions: theory, characterizations and applications. Journal of Data Science, 15(4), 723-740.

69. Yousof, H. M., Altun, E. and Hamedani, G. G. (2018a). A new extension of Frechet distribution with regression models, residual analysis and characterizations. Journal of Data Science, 16(4), 743-770.

70. Yousof, H. M., Altun, E., Rasekhi, M., Alizadeh, M., Hamedani, G. G. and Ali, M. M. (2019b). A new lifetime model with regression models, characterizations and applications. Communications in Statistics-Simulation and Computation, 48(1), 264-286.

71. Yousof, H. M., Altun, E., Ramires, T. G., Alizadeh, M. and Rasekhi, M. (2018). A new family of distributions with properties, regression models and applications. Journal of Statistics and Management Systems, 21, 1, 163-188. 
72. Yousof, H. M., Hamedani, G. G. and Ibrahim, M. (2020). The two-parameter xgamma Fréchet distribution: characterizations, copulas, mathematical properties and different classical estimation methods. Contributions to Mathematics, 2 (2020), 32-41.

73. Yousof, H. M., Majumder, M., Jahanshahi, S. M. A., Ali, M. M. and Hamedani G. G. (2018b). A new Weibull class of distributions: theory, characterizations and applications. Journal of Statistical Research of Iran, 15, 45-83. 